This item was submitted to Loughborough's Research Repository by the author.

Items in Figshare are protected by copyright, with all rights reserved, unless otherwise indicated.

Acoustic scattering in dispersions: improvements in the calculation of single particle scattering coefficients

PLEASE CITE THE PUBLISHED VERSION

http://dx.doi.org/10.1121/1.2737745

PUBLISHER

(c) Acoustical Society of America

VERSION

VoR (Version of Record)

LICENCE

CC BY-NC-ND 4.0

REPOSITORY RECORD

Pinfield, Valerie J.. 2012. "Acoustic Scattering in Dispersions: Improvements in the Calculation of Single Particle Scattering Coefficients”. figshare. https://hdl.handle.net/2134/11083. 
This item was submitted to Loughborough's Institutional Repository (https://dspace.lboro.ac.uk/) by the author and is made available under the following Creative Commons Licence conditions.

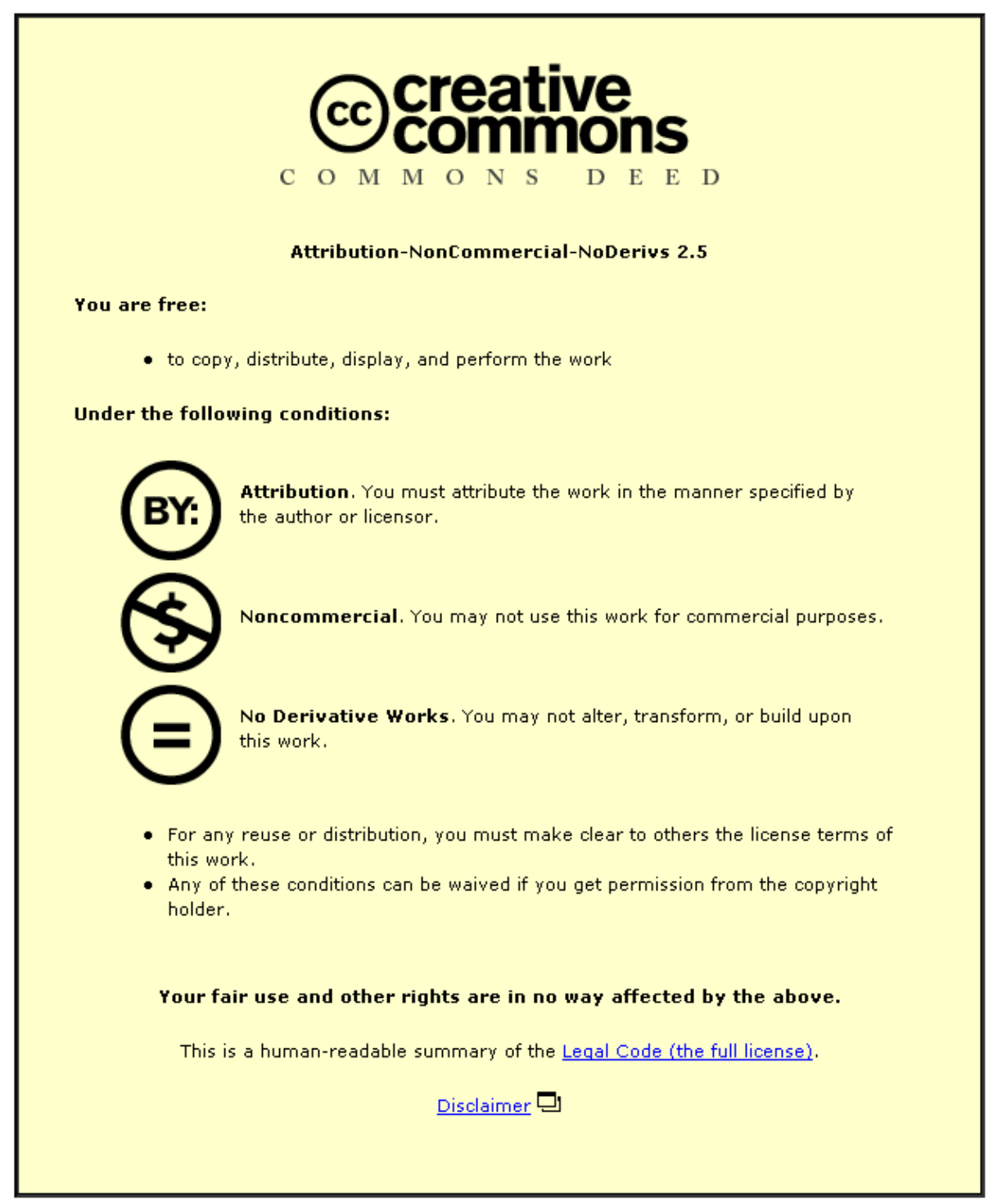

For the full text of this licence, please go to: http://creativecommons.org/licenses/by-nc-nd/2.5/ 


\title{
Acoustic scattering in dispersions: Improvements in the calculation of single particle scattering coefficients
}

\author{
Valerie J. Pinfield ${ }^{\text {a) }}$ \\ Procter Department of Food Science, University of Leeds, Leeds LS2 9JT, United Kingdom
}

(Received 28 November 2006; revised 26 March 2007; accepted 16 April 2007)

\begin{abstract}
Measurements of ultrasound speed and attenuation can be related to the properties of dispersed systems by applying a scattering model. Rayleigh's method for scattering of sound by a spherical object, and its subsequent developments to include viscous, thermal, and other effects (known as the ECAH model) has been widely adopted. The ECAH method has difficulties, including numerical ill-conditioning, calculation of Bessel functions at large arguments, and inclusion of thermal effects in all cases. The present work develops techniques for improving the ECAH calculations to allow its use in instrumentation. It is shown that thermal terms can be neglected in some boundary equations up to $\sim 100 \mathrm{GHz}$ in water, and several simplified solutions result. An analytical solution for the zero-order coefficient is presented, with separate nonthermal and thermal parts, allowing estimation of the thermal contribution. Higher orders have been simplified by estimating the small shear contribution as the inertial limit is approached. The condition of the matrix solutions have been greatly improved by these techniques and by including appropriate scaling factors. A method is presented for calculating the required Bessel functions when the argument is large (high frequency or large particle size). The required number of partial wave orders is also considered. (C) 2007 Acoustical Society of America. [DOI: 10.1121/1.2737745]
\end{abstract}

PACS number(s): 43.35.Bf [RR]

Pages: 205-221

\section{INTRODUCTION}

Ultrasound spectroscopy is used with a wide range of materials in which particles, droplets, or molecules are present in a continuous medium, emulsions, dispersions, gels, and solutions of biomolecules. The propagation speed and attenuation are measured for an ultrasound signal transmitted or reflected through the system. The interaction of ultrasound with the material depends on the contrast between the constituent components. In the simplest form, the contrast is of density and compressibility, causing a change in the ultrasound speed and attenuation in the material. At a more complex level, contributions from thermal contrast, and viscous effects occur, and there may also be absorption due to other physical or chemical processes, such as relaxations of chemical equilibria. Sound speed and attenuation are also affected by the particle size distribution and concentration, and by their spatial distribution, e.g., aggregation.

A number of models exist which attempt to relate the sound speed and attenuation in a system to its physical properties. A substantial review of the approaches to the sound propagation problem for dispersed systems is given by Challis et al., ${ }^{1}$ including coupled phase models, and scattering models. The scattering model treats each particle as a scatterer of sound, and then determines the combined effect of scattering from an ensemble of such particles. The method was developed over a hundred years ago by Rayleigh, ${ }^{2}$ who considered the problem of scattering of sound waves by a single spherical object. Later developments have extended the applicability of the model, for example, to include vis-

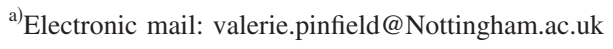

cous effects, compressibility of the scatterer, and thermal effects. The scattering model for spherical objects developed by Epstein and Carhart ${ }^{3}$ and the closely related Allegra and Hawley ${ }^{4}$ model are now well established, and form the basis for the present work. The method is referred to as the ECAH method. This set of models includes viscous and thermal contributions and covers a range of values of the wavenumber-radius parameter $k a$ (where $k$ is the wave number and $a$ the particle radius). The equivalence between the solutions for liquid/liquid (Epstein and Carhart $^{3}$ ), solid/liquid (Allegra and Hawley ${ }^{4}$ ), and solid/solid systems (Ying and Truell ${ }^{5}$ ) has been established (Challis et al. ${ }^{6}$ ), implying that the formulation is of general applicability.

The ECAH method for the scattering of a plane wave by a single spherical particle uses Rayleigh expansions (spherical harmonics) of the sound field potentials, for each wave mode: propagational, thermal, and shear. The appropriate boundary conditions are then applied at the particle surface, resulting in a six square matrix equation to invert for each partial wave order $n$. However, the matrix equation is badly conditioned, causing inaccuracy with the matrix inversion unless a high degree of machine precision is used. The problem of the high precision required for solution was explored by Harlen et al., ${ }^{7}$ and has been documented by Challis and co-workers $\left(\mathrm{O}^{\prime}\right.$ Neill et $\left.a .^{8}{ }^{8}\right)$. Further difficulty arises from the need to calculate spherical Bessel and Hankel functions (Heine functions) at large complex arguments. Such numerical problems have limited the application of the method. Although the ECAH method is applicable to general values of $k a$, both Epstein and Carhart ${ }^{3}$ and Allegra and Hawley ${ }^{4}$ obtained analytical solutions for the lowest order scattering coefficients in the long wavelength limit, where $k a \ll 1$. These results have been more widely used, but restrict the applica- 
tion to a limited frequency and particle size range. At higher values of $k a$, convergence of the sum of scattering coefficients over all wave orders (harmonics) is not monotonic, so that it is difficult to establish how many partial wave orders need to be included. ${ }^{8}$ Some workers have also argued that the inclusion of thermal effects is unnecessary, or that it overcomplicates the solution. ${ }^{9}$

In the work reported here, the objective was to identify simplifications of the ECAH method in order to overcome the problems associated with it. This was achieved by establishing conditions under which certain contributions were small, and therefore causing near-singularity of the matrix. In particular, solutions were obtained for conditions in which the thermal contribution is small, and, independently, conditions for which the viscous contribution is small. In some cases, these contributions can be neglected altogether, whereas in other cases an estimate can be obtained for the small but non-negligible effect. The number of partial wave orders required for accurate velocity and attenuation calculation was also investigated. Additionally, a method was developed for determining the necessary Heine functions at large arguments. The work applies to the determination of the single particle scattering coefficients, and does not consider multiple scattering models. The developments presented here apply to fluid particles in a continuous fluid phase. The set of techniques presented in the paper overcomes many of the current difficulties with the ECAH method. This will permit the use of the scattering model calculations in instrumentation, rather than as a research tool requiring high precision arithmetic.

In Sec. II, the ECAH method is summarized. Section III considers the convergence problem, i.e., the number of partial wave orders needed to achieve a satisfactory result. Modifications to the ECAH method are presented in Sec. IV leading to improved conditioning and a method for calculating Bessel/Hankel functions. Further developments for calculation of the scattering coefficients are given in Sec. V, considering limits in which thermal effects are small, and viscous effects are small. Some numerical results are presented in Sec. VI.

\section{THE ECAH METHOD}

Full details of the ECAH method can be found in the respective publications, Epstein and Carhart ${ }^{3}$ and Allegra and Hawley. ${ }^{4}$ The method solves the single particle scattering coefficient for a single spherical particle in an infinite medium with an incident plane wave. Here, both particle and surrounding medium are assumed to be fluids.

\section{A. Equations for solution}

In summary, the solution is based on a series of steps:

(1) The equations of motion for sound propagation (including continuity of mass, linear Navier-Stokes, energy) are expressed using scalar and vector velocity potentials,

$$
\mathbf{u}=-\boldsymbol{\nabla} \phi+\nabla \times \boldsymbol{\chi},
$$

where $\mathbf{u}$ is the velocity of the fluid, $\phi$ is a scalar potential, and $\boldsymbol{\chi}$ is a vector potential satisfying the condition $\nabla \cdot \boldsymbol{\chi}=0$.
(2) A solution with time dependence of the form $e^{-i \omega t}$ (where $\omega=2 \pi f$ is the angular frequency) is substituted in the equations, giving a Helmholtz equation for each of three wave modes with respective wave numbers

$$
\left(\boldsymbol{\nabla}^{2}+k_{c}^{2}\right) \varphi=0, \quad\left(\boldsymbol{\nabla}^{2}+k_{T}^{2}\right) \psi=0, \quad\left(\boldsymbol{\nabla}^{2}+k_{S}^{2}\right) \boldsymbol{\chi}=\mathbf{0} .
$$

The two scalar potentials correspond to longitudinal propagational and thermal wave modes, while the vector potential represents a transverse shear wave mode. The propagational mode is the "usual" mode by which sound travels in a fluid, whilst the thermal mode represents heat flow and the shear mode arises from viscous effects. Both the thermal and shear modes are highly localized because their amplitude decreases exponentially with each wavelength traveled. The total scalar potential is the sum of the propagational and thermal wave potentials. The axis of propagation of the plane wave is taken as the $z$ axis so that the vector potential reduces to a single component $\chi_{\psi}$ denoted simply $\chi$. The vector potential in Epstein and Carhart ${ }^{3}$ used the symbol $A$; here $\chi$ has been used to avoid confusion with the scattering coefficient $A_{n}$.

(3) The solutions of the Helmholtz equation appropriate for a spherical boundary are partial wave solutions (or Rayleigh series), using spherical harmonic functions (in spherical coordinates), i.e., spherical Bessel or Hankel functions for the radial dependence, and Legendre polynomials (or associated Legendre polynomials) for the angular dependence. The solution is a sum over each partial wave order, so that, for example

$$
\varphi=\sum_{n=0}^{\infty} i^{n}(2 n+1) A_{n} h_{n}\left(k_{c} r\right) P_{n}(\cos \theta) .
$$

(4) Continuity conditions are applied at the boundary for velocity, stress, and thermal effects. A set of six boundary conditions must be solved for each partial wave order $n$ to determine the scattering coefficient $A_{n}$.

\section{B. The wave modes}

The wave numbers, $k_{c}$ for the propagational mode, $k_{T}$ for the thermal mode, and $k_{S}$ for the shear mode, are given to a very good approximation in fluids by

$$
\begin{aligned}
& k_{c}=\frac{\omega}{\nu}+i \alpha, \quad k_{T}=\left(\frac{\omega}{2 \sigma}\right)^{1 / 2}(1+i), \\
& k_{S}=\left(\frac{\omega}{2 v}\right)^{1 / 2}(1+i),
\end{aligned}
$$

where $\nu$ is the speed of sound, $\alpha$ is the attenuation, $\sigma$ the thermal diffusivity, such that $\sigma=\tau / \rho C_{p}$ where $\tau$ is the thermal conductivity, $\rho$ is the density, $C_{p}$ is the specific heat capacity at constant pressure, and $v=\eta / \rho$ is the kinematic viscosity, where $\eta$ is shear viscosity.

The solution forms for the various wave potentials are given in the following, using the Hankel function $h_{n}$ outside the particle, and the spherical Bessel function $j_{n}$ inside the particle. Primed quantities refer to the inside of the particle, 
and subscripts $0, c, T$, and $S$ refer to the incident wave, propagational, thermal, and shear modes, respectively, throughout the paper,

$$
\begin{aligned}
& \varphi=\sum_{n=0}^{\infty} i^{n}(2 n+1) A_{n} h_{n}\left(k_{c} r\right) P_{n}(\cos \theta), \\
& \psi=\sum_{n=0}^{\infty} i^{n}(2 n+1) B_{n} h_{n}\left(k_{T} r\right) P_{n}(\cos \theta), \\
& \chi=\sum_{n=1}^{\infty} i^{n}(2 n+1) C_{n} h_{n}\left(k_{S} r\right) P_{n}^{1}(\cos \theta), \\
& \varphi^{\prime}=\sum_{n=0}^{\infty} i^{n}(2 n+1) A_{n}^{\prime} j_{n}\left(k_{c}^{\prime} r\right) P_{n}(\cos \theta), \\
& \psi^{\prime}=\sum_{n=0}^{\infty} i^{n}(2 n+1) B_{n}^{\prime} j_{n}\left(k_{T}^{\prime} r\right) P_{n}(\cos \theta), \\
& \chi^{\prime}=\sum_{n=1}^{\infty} i^{n}(2 n+1) C_{n}^{\prime} j_{n}\left(k_{S}^{\prime} r\right) P_{n}^{1}(\cos \theta) .
\end{aligned}
$$

Similarly the incident field (a plane wave) can be expressed as

$$
\varphi_{0}=\sum_{n=0}^{\infty} i^{n}(2 n+1) j_{n}\left(k_{c} r\right) P_{n}(\cos \theta) .
$$

\section{Boundary conditions}

At the surface of the spherical particle, the boundary equations result from the requirement for continuity of fluid velocity components $u_{r}$ and $u_{\theta}$, the stress components $P_{r r}$ and $P_{r \theta}$, temperature $T$, and heat flux $\tau \partial T / \partial r$. These conditions are applied at the spherical boundary $r=a$ where $a$ is the radius of the particle.

For any scalar potential $\phi$ (propagational and thermal modes) the components are given by

$$
\begin{aligned}
& u_{r}=-\frac{\partial \phi}{\partial r}, \quad u_{\theta}=-\frac{1}{r} \cdot \frac{\partial \phi}{\partial \theta}, \\
& P_{r r}=\left(i \omega \rho-2 \eta k^{2}\right) \phi-2 \eta \frac{\partial^{2} \phi}{\partial r^{2}}, \\
& P_{r \theta}=2 \eta \frac{\partial}{\partial \theta}\left(\frac{\phi}{r^{2}}-\frac{1}{r} \frac{\partial \phi}{\partial r}\right), \\
& T=\Gamma \phi,
\end{aligned}
$$

where the physical properties, wave number $k$, and thermal factor $\Gamma$ are appropriate to the wave mode and the material.

The thermal factors are given by

$$
\Gamma_{c}=\frac{-i k_{c}^{2}(\gamma-1)}{\beta\left(\omega+i \gamma \sigma k_{c}^{2}\right)} \approx \frac{k_{c}^{2}(\gamma-1)}{\beta \sigma k_{T}^{2}},
$$

$$
\Gamma_{T}=\frac{-i k_{T}^{2}(\gamma-1)}{\beta\left(\omega+i \gamma \sigma k_{T}^{2}\right)} \approx-\frac{1}{\beta \sigma},
$$

where $\gamma=C_{p} / C_{\nu}$ is the ratio of the specific heat capacities and $\beta$ is the thermal expansivity. The subscripts $c, T$, and $S$ refer throughout to the propagational, thermal, and shear modes, respectively. The approximate results on the righthand side are valid under the condition

$$
\left|\frac{k_{c}^{2}}{k_{T}^{2}}\right| \approx \frac{\omega \sigma}{\nu^{2}} \ll 1,
$$

which is true over a very wide frequency range (up to $\sim 10^{11} \mathrm{~Hz}$ for water at $30{ }^{\circ} \mathrm{C}$ ).

It is worth noting that the ratio of the temperature factors for the propagational and thermal modes is very small, showing that the temperature changes are dominated by the thermal mode,

$$
\left|\frac{\Gamma_{c}}{\Gamma_{T}}\right| \approx \frac{k_{c}^{2}(\gamma-1)}{k_{T}^{2}},
$$

which is very small.

For the shear wave mode,

$$
\begin{aligned}
& u_{r}=\frac{1}{r \sin \theta} \frac{\partial}{\partial \theta}(\chi \sin \theta), \quad u_{\theta}=-\frac{1}{r} \frac{\partial}{\partial r}(r \chi), \\
& P_{r r}=\frac{2 \eta}{\sin \theta} \frac{\partial}{\partial \theta}\left[\sin \theta\left(-\frac{\chi}{r^{2}}+\frac{1}{r} \frac{\partial \chi}{\partial r}\right)\right], \\
& P_{r \theta}=\eta\left[\left(\frac{2 \chi}{r^{2}}-\frac{\partial^{2} \chi}{\partial r^{2}}\right)+\frac{1}{r^{2}} \frac{\partial}{\partial \theta}\left(\frac{1}{\sin \theta} \frac{\partial}{\partial \theta}(\chi \sin \theta)\right)\right] .
\end{aligned}
$$

Equations (14) and (15) for stress are appropriate for fluids. The $P_{r r}$ component is usually divided by the factor $i \omega \rho$ in the boundary equations, and the $P_{r \theta}$ by a factor of $\eta$. Further details on the stress relations are given by Povey (Ref. 10, pp. 106-109), and also by Epstein and Carhart ${ }^{3}$ and Allegra and Hawley. ${ }^{4}$ Here, bulk viscosity has been neglected. The shear wave modes do not contribute to the temperature or heat flow.

When evaluating the boundary conditions, the following relations which were listed by Epstein and Carhart ${ }^{3}$ are useful:

$$
\begin{aligned}
& -\frac{d P_{n}(\cos \theta)}{d \theta}=P_{n}^{1}(\cos \theta), \\
& \frac{1}{\sin \theta} \frac{d}{d \theta}\left(\sin \theta P_{n}^{1}(\cos \theta)\right)=n(n+1) P_{n}(\cos \theta) .
\end{aligned}
$$

\section{Multiple scattering}

In practice, ultrasound measurements are made for a system or ensemble of particles in a dispersion. Usually, the ultrasound velocity (or speed) and/or attenuation is measured, often over a range of frequencies. To relate the single particle scattering properties derived in the subsequent sec- 
tions to the sound speed and attenuation in a dispersion, a scattering theory is used. The limiting far-field solution has the form

$$
\varphi \rightarrow \frac{e^{i k_{c} r}}{r} f(\theta),
$$

where

$$
f(\theta)=\frac{1}{i k_{c}} \sum_{n=0}^{\infty}(2 n+1) A_{n} P_{n}(\cos \theta) .
$$

The multiple scattering result for the wave number of the dispersion, $K$ (Refs. 11-13) is

$$
\begin{aligned}
\left(\frac{K}{k_{c}}\right)^{2}= & +\frac{3 \phi}{k_{c}^{2} a^{3}} f(0)+\frac{9 \phi^{2}}{4 k_{c}^{4} a^{6}}\left(f^{2}(\pi)-f^{2}(0)\right. \\
& \left.-\int_{0}^{\pi} d \theta \frac{1}{\sin (\theta / 2)}\left(\frac{d}{d \theta} f^{2}(\theta)\right)\right) .
\end{aligned}
$$

Note that here the symbol $\phi$ refers to the volume fraction of the dispersed particles.

The resulting dominant terms in the single scattering result are as follows:

$$
\begin{aligned}
\frac{1}{\nu_{\text {Soln }}^{2}} & =\frac{1}{\nu^{2}}\left[1+\frac{3 \phi}{k_{c}^{3} a^{3}} \sum_{n=0}(2 n+1) \operatorname{Im}\left(A_{n}\right)\right], \\
\alpha_{\text {soln }} & =\alpha-\frac{3 \phi}{2 k_{c}^{2} a^{3}} \sum_{n=0}(2 n+1) \operatorname{Re}\left(A_{n}\right) .
\end{aligned}
$$

Hence it can be seen that the first-order term in the velocity and attenuation relates to an infinite sum of the scattering coefficients $(2 n+1) A_{n}$.

Having summarized the ECAH method, the following sections examine a number of methods developed to improve the numerical calculation resulting from the formulation. First, the number of partial wave orders needed in the infinite sum is considered.

\section{NUMBER OF PARTIAL WAVE ORDERS: CONVERGENCE}

The scattered field for the propagational mode depends on an infinite sum of the scattering coefficients over all partial wave orders. It is this field which is detected experimentally and for which the velocity and attenuation are measured. When calculating the scattered field, the series must be truncated at a finite wave order. In the long wavelength region (small $k_{c} a$ ), where the model has been extensively applied, only the lowest orders make a significant contribution, so that the calculation may be limited to $n=0,1,2$. However, at larger values of $k_{c} a$, higher orders become more significant and must be included in the calculated scattered field. It is necessary to determine at what order the series can be terminated without significant loss of accuracy.

O'Neill et al. ${ }^{8}$ explored the convergence of the series, demonstrating that the contribution of successive scattering coefficients does not decrease monotonically. The sum shows oscillatory behavior, and some plateaus at which convergence might be believed to have been achieved, although subsequent orders are significant. They demonstrated that where the product $k_{c} a$ is large, many tens or even over a hundred terms in the series may be necessary to obtain a sufficiently accurate solution. An empirical result was fitted to the data, relating the maximum number of partial wave orders required to the wave number parameter $k_{c} a$, thus

$$
n \operatorname{Max}=1.05 k_{c} a+4
$$

(limited to particle sizes above $1 \mu \mathrm{m}$ in diameter).

O'Neill et al. ${ }^{8}$ considered the solution for the scattering coefficients and their sum in the scattered field. However, the magnitude of the scattered wave field for each partial wave order will be limited by the corresponding magnitude of the incoming wave field for that same order. The scattering process, including thermal and shear effects, will modify the proportion of the incident field of a particular order which is scattered as propagational mode, but the magnitude is limited by the incoming wave. It should be noted that this is a linear formulation, and there is no conversion between modes of different orders. The incoming plane wave potential was given in Eq. (6). Each partial wave order has an incident amplitude at the particle surface proportional to the Bessel function $j_{n}\left(k_{c} a\right)$, and this is the limiting value of the outgoing "amplitude." The scattered propagational field [Eq. (5)] has an amplitude at the surface proportional to $h_{n}\left(k_{c} a\right)$ so a measure of the maximum coefficient value in heuristic terms is the ratio $j_{n}\left(k_{c} a\right) / h_{n}\left(k_{c} a\right)$.

The functions $j_{n}(z), h_{n}(z)$, and the ratio $j_{n}(z) / h_{n}(z)$ are plotted against order $n$ for a range of values of a real argument $z$ (Fig. 1). For real arguments, $j_{n}(z)$ is real, but $h_{n}(z)$ is complex, and the ratio is plotted as the absolute value. It can be seen from the plots that all functions undergo a dramatic "termination" when the order $n$ reaches a value just greater than the argument $z$. The Bessel function $j_{n}(z)$ stops its oscillatory behavior and becomes negligible above this order, the Hankel function $h_{n}(z)$ explodes to very large values, and the absolute value of the ratio $j_{n}(z) / h_{n}(z)$ suddenly stops being oscillatory and becomes negligible thereafter (i.e., for all higher orders). The point at which these changes occur relate closely to the empirical formula obtained by O'Neill et al. ${ }^{8}$ That is around

$$
n \sim k_{c} a+4 .
$$

The plots demonstrate that the limit of partial wave orders is determined by the incident wave magnitude for the various orders which limits the amount of scattered field of the same order. Hence the number of partial wave orders required is defined by the number of wave orders needed to describe the incident field for a particle of a given radius. For a plane wave of very large wavelength compared with the particle radius, a small number of partial wave modes are sufficient to define the incident field at the particle surface, since it varies very little across the particle. At smaller wavelengths, more and more wave modes are needed to define the plane wave as partial wave modes at the particle surface. When the wavelength is shorter than the particle radius, for example $\left(k_{c} a>1\right)$, many modes are needed since the wave amplitude is changing rapidly across the surface. The higher 


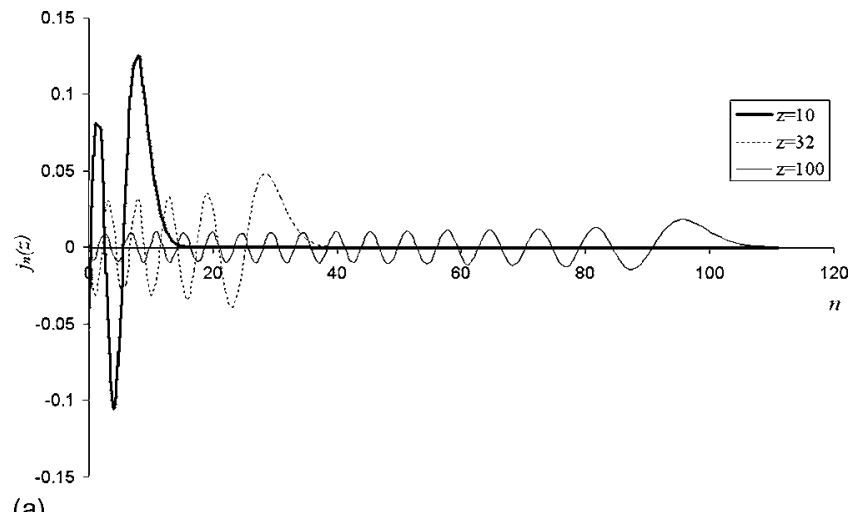

(a)

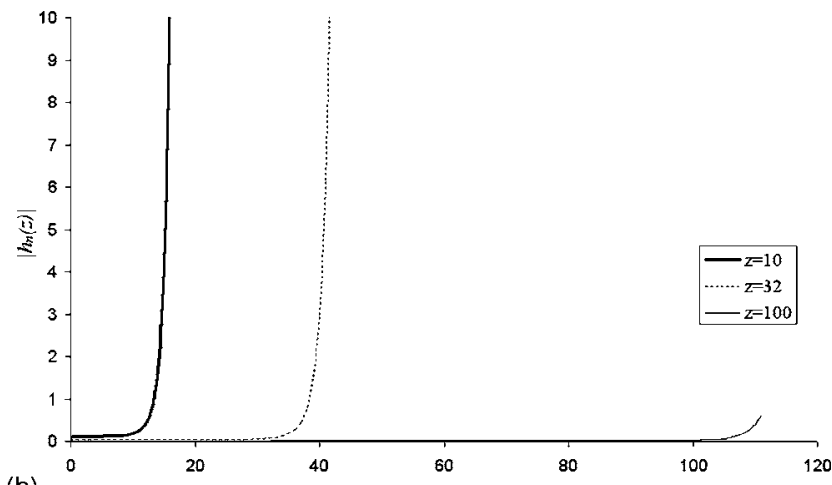

(b)

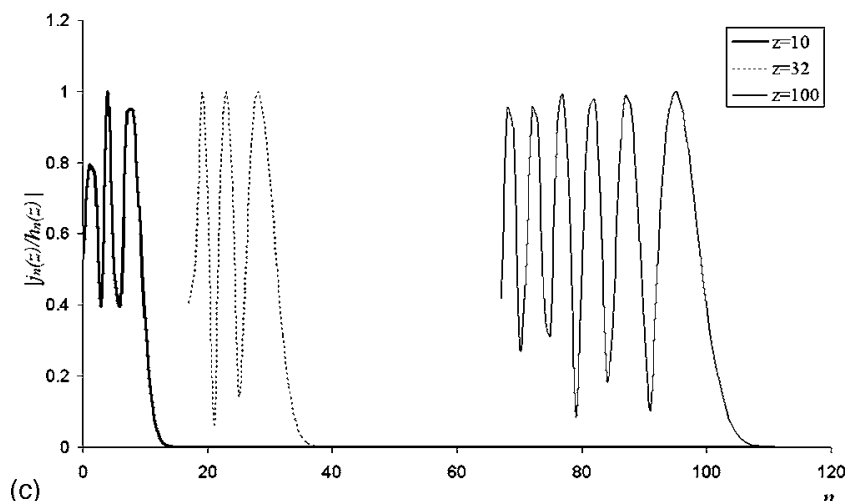

FIG. 1. Plots of the Bessel functions for the incoming and outgoing fields as a function of partial wave order $n$ for a range of real arguments $z$. (a) $j_{n}(z)$, (b) $h_{n}(z)$, and (c) $j_{n}(z) / h_{n}(z)$. In (c) the oscillatory behavior over the full range of $n$ has been omitted for clarity. The plots show that the number of orders for which there is a nonzero incoming partial wave is a little greater than the value of $z$; at higher orders $n$ (on the $x$ axis) the function remains zero. Hence the number of orders needed for a scattering calculation is a little more than $k_{c} a$. In (c) much of the oscillatory behavior for both $z=32$ and $z=100$ has been omitted for clarity.

wave orders allow greater "detail" in the variation of the incident field, which are required as the wavelength becomes shorter.

\section{MODIFICATIONS TO ECAH METHOD}

One of the main difficulties with the ECAH method is that the set of boundary equations is not well suited to numerical solution. The equations form a $6 \times 6$ matrix equation for each order $n$ which is inverted to obtain the six scattering coefficients. For the case $n=0$ the system is only a $4 \times 4$ matrix. The Hankel functions and Bessel functions used outside and inside the particle, respectively, diverge in magni- tude, one becomes small, the other very large as the argument increases. Thus the matrix elements become similarly disparate in magnitude, rendering inversion inaccurate. The quality of the inversion is usually measured by the "condition number" of the matrix, which when multiplied by the machine precision gives the accuracy of the result. The condition numbers are typically very large, especially at large frequencies. A second difficulty is that determination of the Bessel functions is difficult and inaccurate for large arguments. These two elements are addressed in the following sections. The work concentrates on high frequencies or large particle sizes where these problems are most acute. In the long wavelength region (where $k_{c} a \ll 1$ ) analytical solutions are available and these problems do not arise.

\section{A. Scaling of thermal and shear wave mode potentials}

A straightforward scaling in the wave potential definitions has been used to address the problem of the widely differing magnitude of the components in the boundary equations. The problem occurs primarily for the thermal and shear wave modes, and it is these modes to which the scaling has been applied.

The wave potentials have been redefined with a factor of the relevant Bessel and Hankel function evaluated at the particle surface in each partial wave order. Bessel function zeros occur only for real arguments, so for arguments of the form $s(1+i)$ there are no zeros. Both thermal and shear wave numbers take this form, so that division by the Bessel function is appropriate. Thus the wave potentials are redefined as

$$
\begin{aligned}
& \psi=\sum_{n=0}^{\infty} i^{n}(2 n+1) B_{n} P_{n}(\cos \theta) h_{n}\left(k_{T} r\right) / h_{n}\left(k_{T} a\right), \\
& \chi=\sum_{n=1}^{\infty} i^{n}(2 n+1) C_{n} P_{n}^{1}(\cos \theta) h_{n}\left(k_{S} r\right) / h_{n}\left(k_{S} a\right), \\
& \psi^{\prime}=\sum_{n=0}^{\infty} i^{n}(2 n+1) B_{n}^{\prime} P_{n}(\cos \theta) j_{n}\left(k_{T}^{\prime} r\right) / j_{n}\left(k_{T}^{\prime} a\right), \\
& \chi^{\prime}=\sum_{n=1}^{\infty} i^{n}(2 n+1) C_{n}^{\prime} P_{n}^{1}(\cos \theta) j_{n}\left(k_{S}^{\prime} r\right) / j_{n}\left(k_{S}^{\prime} a\right) .
\end{aligned}
$$

The coefficients for the thermal and shear modes are not now the same as those in ECAH, since they include the appropriate factor.

\section{B. Stress}

The adjusted stress components are given here, including the scaling factors shown earlier for the thermal and shear modes. The stress terms then have ratios of the Bessel function derivatives with the scaling Bessel function. The stress components $P_{r r}$ at the boundary have been divided by the factor $i \omega \rho$ (which is equal to $\eta k_{S}^{2}$ ) to define the parameters in the following. For convenience, the inertial parts of the stress (the first terms in $q$ ) have been separated from the viscous parts, $w$, 


$$
\begin{aligned}
& q_{0 n}=j_{n}\left(k_{c} a\right)+w_{0 n}, \\
& q_{c n}=h_{n}\left(k_{c} a\right)+w_{c n}, \\
& q_{T n}=1+w_{T n}, \\
& q_{c n}^{\prime}=\hat{\rho} j_{n}\left(k_{c}^{\prime} a\right)+w_{c n}^{\prime}, \\
& q_{T n}^{\prime}=\hat{\rho}+w_{T n}^{\prime} .
\end{aligned}
$$

where the terms denoted $w$ represent the shear or viscous contribution to the stress which are given by

$$
\begin{aligned}
& w_{0 n}=-2 \frac{\left(k_{c} a\right)^{2}}{\left(k_{S} a\right)^{2}}\left[j_{n}\left(k_{c} a\right)+j_{n}^{\prime \prime}\left(k_{c} a\right)\right], \\
& w_{c n}=-2 \frac{\left(k_{c} a\right)^{2}}{\left(k_{S} a\right)^{2}}\left[h_{n}\left(k_{c} a\right)+h_{n}^{\prime \prime}\left(k_{c} a\right)\right], \\
& w_{T n}=-2 \frac{\left(k_{T} a\right)^{2}}{\left(k_{S} a\right)^{2}}\left[1+h_{n}^{\prime \prime}\left(k_{T} a\right) / h_{n}\left(k_{T} a\right)\right], \\
& w_{c n}^{\prime}=-2 \hat{\rho} \frac{\left(k_{c}^{\prime} a\right)^{2}}{\left(k_{S}^{\prime} a\right)^{2}}\left[j_{n}\left(k_{c}^{\prime} a\right)+j_{n}^{\prime \prime}\left(k_{c}^{\prime} a\right)\right], \\
& w_{T n}^{\prime}=-2 \hat{\rho} \frac{\left(k_{T}^{\prime} a\right)^{2}}{\left(k_{S}^{\prime} a\right)^{2}}\left[1+j_{n}^{\prime \prime}\left(k_{T}^{\prime} a\right) / j_{n}\left(k_{T}^{\prime} a\right)\right] .
\end{aligned}
$$

For the shear waves,

$$
\begin{aligned}
& q_{S n}=\frac{2 n(n+1)}{\left(k_{S} a\right)^{2}}\left[k_{S} a \frac{h_{n}^{\prime}\left(k_{S} a\right)}{h_{n}\left(k_{S} a\right)}-1\right], \\
& q_{S n}^{\prime}=\frac{2 n(n+1) \hat{\rho}}{\left(k_{S}^{\prime} a\right)^{2}}\left[k_{S}^{\prime} a \frac{j_{n}^{\prime}\left(k_{S}^{\prime} a\right)}{j_{n}\left(k_{S}^{\prime} a\right)}-1\right] .
\end{aligned}
$$

Note that primed and double primed Bessel and Hankel functions denote first and second derivatives rather than the inside of the particle.

For the other stress component, $P_{r \theta}$, the stress is divided by a common factor of $\eta$, thus

$$
\begin{aligned}
& d_{0 n}=\left[k_{c} a j_{n}^{\prime}\left(k_{c} a\right)-j_{n}\left(k_{c} a\right)\right], \\
& d_{c n}=\left[k_{c} a h_{n}^{\prime}\left(k_{c} a\right)-h_{n}\left(k_{c} a\right)\right], \\
& d_{T n}=\left[k_{T} a h_{n}^{\prime}\left(k_{T} a\right) / h_{n}\left(k_{T} a\right)-1\right], \\
& d_{c n}^{\prime}=\hat{\eta}\left[k_{c}^{\prime} a j_{n}^{\prime}\left(k_{c}^{\prime} a\right)-j_{n}\left(k_{c}^{\prime} a\right)\right], \\
& d_{T n}^{\prime}=\hat{\eta}\left[k_{T}^{\prime} a j_{n}^{\prime}\left(k_{T}^{\prime} a\right) / j_{n}\left(k_{T}^{\prime} a\right)-1\right]
\end{aligned}
$$

and for the shear waves

$$
\begin{aligned}
& d_{S n}=\frac{1}{2}\left[\left(k_{S} a\right)^{2} \frac{h_{n}^{\prime \prime}\left(k_{S} a\right)}{h_{n}\left(k_{S} a\right)}+\left(n^{2}+n-2\right)\right], \\
& d_{S n}^{\prime}=\hat{\eta} \cdot \frac{1}{2}\left[\left(k_{S}^{\prime} a\right)^{2} \frac{j_{n}^{\prime \prime}\left(k_{S}^{\prime} a\right)}{j_{n}\left(k_{S}^{\prime} a\right)}+\left(n^{2}+n-2\right)\right] .
\end{aligned}
$$

\section{Special case $n=1$}

In the particular case when $n=1$, some of these expressions become inaccurate, since they result in a small difference between two much larger quantities when the argument is small. Greater accuracy is achieved using the general Bessel function relation

$$
z R_{n}^{\prime}(z)-n R_{n}(z)=-z R_{n+1}(z)
$$

where $R_{n}$ denotes any (spherical) Bessel function. Thus the stress terms become

$$
\begin{aligned}
& q_{S 1}=-\frac{4 h_{2}\left(k_{S} a\right)}{\left(k_{S} a\right) h_{1}\left(k_{S} a\right)}, \\
& q_{S 1}^{\prime}=-\frac{4 \hat{\rho} j_{2}\left(k_{S}^{\prime} a\right)}{\left(k_{S}^{\prime} a\right) j_{1}\left(k_{S}^{\prime} a\right)}, \\
& d_{01}=-k_{c} a j_{2}\left(k_{c} a\right), \\
& d_{c 1}=-k_{c} a h_{2}\left(k_{c} a\right), \\
& d_{c 1}^{\prime}=-\hat{\eta}_{c}^{\prime} a j_{2}\left(k_{c}^{\prime} a\right), \\
& d_{T 1}=-k_{T} a h_{2}\left(k_{T} a\right) / h_{1}\left(k_{T} a\right), \\
& d_{T 1}^{\prime}=-\hat{\eta} k_{T}^{\prime} a j_{2}\left(k_{T}^{\prime} a\right) / j_{1}\left(k_{T} a\right) .
\end{aligned}
$$

\section{Bessel functions at large arguments}

As has been stated previously, another difficulty with the ECAH method is the requirement to evaluate the spherical Bessel and Hankel functions with large complex arguments. In particular, the values of the thermal and shear parameters $k_{T} a, k_{T}^{\prime} a, k_{S} a, k_{S}^{\prime} a$ can be very large, and have equal real and imaginary parts. The imaginary term is especially a problem for the Bessel functions. The issue has been partly addressed by taking the ratio with the Bessel function itself in the shear and thermal wave modes, as seen in the preceding section. In so doing, the need to evaluate the Bessel functions themselves is removed, leaving only ratios of the derivatives of the Bessel function with its zero-order derivative at the boundary, e.g., $j_{n}^{\prime}\left(k_{T}^{\prime} a\right) / j_{n}\left(k_{T} a\right)$. At moderate values of the argument, this ratio would be evaluated numerically in the usual way, by determining the value of the derivative and the function itself. However, when the argument is large, it is appropriate to find an alternative way of calculating the ratio. For this reason, analytical approximations to the relevant ratios were sought for the case when the arguments are large.

The following recurrence relations apply to both types of Bessel function: 


$$
z R_{n}^{\prime}(z)+(n+1) R_{n}(z)=z R_{n-1}(z)
$$

and

$$
z^{2} R_{n}^{\prime \prime}(z)+2 z R_{n-1}(z)=-z^{2} R_{n}(z)+(n+1)(n+2) R_{n}(z),
$$

where $R_{n}$ denotes the appropriate Bessel function, $j_{n}$ or $h_{n}$. Note that the second of these equations [Eq. (34)] is given incorrectly in Epstein and Carhart. ${ }^{3}$ Dividing throughout to obtain the required ratios leads to

$$
\begin{aligned}
& \frac{R_{n}^{\prime}(z)}{R_{n}(z)}=\frac{R_{n-1}(z)}{R_{n}(z)}-\frac{(n+1)}{z}, \\
& \frac{R_{n}^{\prime \prime}(z)}{R_{n}(z)}=-1+\frac{(n+1)(n+2)}{z^{2}}-\frac{2 R_{n-1}(z)}{z R_{n}(z)} .
\end{aligned}
$$

The series expansion of the spherical Hankel function gives, when $z$ is large,

$$
\frac{h_{n-1}(z)}{h_{n}(z)}=i+\frac{n}{z}+O\left(\frac{1}{z^{2}}\right)
$$

and hence the derivative ratios are given by

$$
\begin{aligned}
& \frac{h_{n}^{\prime}(z)}{h_{n}(z)}=i-\frac{1}{z}+O\left(\frac{1}{z^{2}}\right), \\
& \frac{h_{n}^{\prime \prime}(z)}{h_{n}(z)}=-1-\frac{2 i}{z}+O\left(\frac{1}{z^{2}}\right), \\
& \frac{h_{2}(z)}{h_{1}(z)}=-i+\frac{2}{z}+O\left(\frac{1}{z^{2}}\right) .
\end{aligned}
$$

Inside the particle, the spherical Bessel function ratios are needed. The limiting form of the function is oscillatory, but the arguments are of the form

$$
z=(1+i)|z| / \sqrt{2}
$$

for the thermal and shear wave modes. Hence when the function is written in exponential form, the dominant term when $|z|$ is large is that including the factor $e^{|z|}$, so that

$$
j_{n}(z) \approx-\frac{i^{n}}{2 i z} e^{-i z} .
$$

However, a greater accuracy is needed than is given by this limiting form, so that the result was determined numerically from calculated Bessel function values to obtain.

$$
\frac{j_{n-1}(z)}{j_{n}(z)}=-i+\frac{n}{z}+O\left(\frac{1}{z^{2}}\right)
$$

and thus the derivatives are given by

$$
\begin{aligned}
& \frac{j_{n}^{\prime}(z)}{j_{n}(z)}=-i-\frac{1}{z}+O\left(\frac{1}{z^{2}}\right), \\
& \frac{j_{n}^{\prime \prime}(z)}{j_{n}(z)}=-1+\frac{2 i}{z}+O\left(\frac{1}{z^{2}}\right),
\end{aligned}
$$

$$
\frac{j_{2}(z)}{j_{1}(z)}=i+\frac{2}{z}+O\left(\frac{1}{z^{2}}\right)
$$

These are valid also for $n=0$.

When the shear or thermal wave number parameters are large, the above presented forms of the relevant Bessel function ratios can be used, thus avoiding the need to calculate Bessel functions for large complex arguments. The method was made possible by scaling all shear and thermal terms by the relevant Bessel function evaluated at the particle boundary.

\section{Equations for solution}

The resulting six boundary equations for each partial wave order are given in the following, using the notation defined in the previous sections. These equations are in the order $u_{r}, P_{r r}, u_{\theta}, P_{r \theta}$, temperature, and heat flow. The thermal and shear wave modes have the Bessel function scaling factors as included in the potential definitions presented earlier. These differ from the ECAH method,

$$
\begin{gathered}
k_{c} a j_{n}^{\prime}\left(k_{c} a\right)+k_{c} a h_{n}^{\prime}\left(k_{c} a\right) A_{n}+k_{T} a \frac{h_{n}^{\prime}\left(k_{T} a\right)}{h_{n}\left(k_{T} a\right)} B_{n}-n(n+1) C_{n} \\
=k_{c}^{\prime} a j_{n}^{\prime}\left(k_{c}^{\prime} a\right) A_{n}^{\prime}+k_{T}^{\prime} a \frac{j_{n}^{\prime}\left(k_{T}^{\prime} a\right)}{j_{n}\left(k_{T}^{\prime} a\right)} B_{n}^{\prime}-n(n+1) C_{n}^{\prime}, \\
q_{0 n}+q_{c n} A_{n}+q_{T n} B_{n}+q_{S n} C_{n}=q_{c n}^{\prime} A_{n}^{\prime}+q_{T n}^{\prime} B_{n}^{\prime}+q_{S n}^{\prime} C_{n}^{\prime}, \\
j_{n}\left(k_{c} a\right)+h_{n}\left(k_{c} a\right) A_{n}+B_{n}-\left[1+k_{S} a \frac{h_{n}^{\prime}\left(k_{S} a\right)}{h_{n}\left(k_{S} a\right)}\right] C_{n} \\
=j_{n}\left(k_{c}^{\prime} a\right) A_{n}^{\prime}+B_{n}^{\prime}-\left[1+k_{S}^{\prime} \frac{j_{n}^{\prime}\left(k_{S}^{\prime} a\right)}{j_{n}\left(k_{S}^{\prime} a\right)}\right] C_{n}^{\prime}, \\
d_{0 n}+d_{c n} A_{n}+d_{T n} B_{n}-d_{S n} C_{n}=d_{c n}^{\prime} A_{n}^{\prime}+d_{T n}^{\prime} B_{n}^{\prime}-d_{S n}^{\prime} C_{n}^{\prime},
\end{gathered}
$$

$$
\Gamma_{c} j_{n}\left(k_{c} a\right)+\Gamma_{c} h_{n}\left(k_{c} a\right) A_{n}+\Gamma_{T} B_{n}=\Gamma_{c}^{\prime} j_{n}\left(k_{c}^{\prime} a\right) A_{n}^{\prime}+\Gamma_{T}^{\prime} B_{n}^{\prime},
$$

$$
\begin{gathered}
\Gamma_{c} k_{c} a j_{n}^{\prime}\left(k_{c} a\right)+\Gamma_{c} k_{c} a h_{n}^{\prime}\left(k_{c} a\right) A_{n}+\Gamma_{T} k_{T} a \frac{h_{n}^{\prime}\left(k_{T} a\right)}{h_{n}\left(k_{T} a\right)} B_{n} \\
=\hat{\tau}\left\{\Gamma_{c}^{\prime} k_{c}^{\prime} a j_{n}^{\prime}\left(k_{c}^{\prime} a\right) A_{n}^{\prime}+\Gamma_{T}^{\prime} k_{T}^{\prime} a \frac{j_{n}^{\prime}\left(k_{T}^{\prime} a\right)}{j_{n}\left(k_{T}^{\prime} a\right)} B_{n}^{\prime}\right\} .
\end{gathered}
$$

The solution has been made numerically more stable, and a method has been established to avoid determining Bessel functions for large arguments. Further improvements are possible in certain conditions, in which some wave modes make a small or negligible contribution to the scattering. These simplifications are considered in the following sections. 


\section{SIMPLIFICATIONS FOR THE ECAH METHOD}

\section{A. Solution for $\boldsymbol{A}_{0}$}

\section{Equations for the limit $k_{c}^{2} / k_{T}^{2} \ll 1$}

When $k_{c}^{2} / k_{T}^{2} \ll 1$ (up to $10^{11} \mathrm{~Hz}$ in water) it is also true that the ratio of the thermal factors for the propagational and thermal modes is small (see Sec. II C), $\Gamma_{c} / \Gamma_{T} \ll 1$. Physically, this means that the temperature change caused by the propagational mode is much smaller than that produced by a thermal mode of the same displacement amplitude. Since the thermal modes are produced at the boundary of the particle in order to ensure continuity of temperature and heat flux, only a small "amount" of thermal mode is needed to compensate for a relatively "large" temperature difference caused by the propagational modes inside and outside the particle.

For the zero-order solution, only four boundary conditions are applicable, Eqs. (42), (43), (46), and (47). Comparing Eqs. (42) and (47), the propagational mode terms in the heat flux equation (47) are a ratio $\Gamma_{c} / \Gamma_{T}$ smaller than the thermal terms. Hence Eq. (47) is satisfied almost entirely by the thermal modes alone, leading to the condition

$$
B_{0}^{\prime}=\frac{\tau \Gamma_{T}}{\tau^{\prime} \Gamma_{T}^{\prime}} \cdot \frac{k_{T} a}{k_{T}^{\prime} a} \cdot \frac{h_{0}^{\prime}\left(k_{T} a\right)}{h_{0}\left(k_{T} a\right)} \frac{j_{0}\left(k_{T}^{\prime} a\right)}{j_{0}^{\prime}\left(k_{T}^{\prime} a\right)} B_{0} .
$$

This approximation was identified by Epstein and Carhart ${ }^{3}$ when obtaining limiting solutions for the long wavelength limit. However, their conclusions are also valid for general values of $k_{c} a$ and therefore can be applied generally, depending only on the smallness of the ratio $k_{c}^{2} / k_{T}^{2}$. Here the solution is continued for the general case, rather than taking the long wavelength limit. Equation (48) can be substituted into the other three boundary equations (42), (43), and (46) to obtain:

$$
\begin{aligned}
& k_{c} a h_{0}^{\prime}\left(k_{c} a\right) A_{0}+b_{1} B_{0}-k_{c}^{\prime} a j_{0}^{\prime}\left(k_{c}^{\prime} a\right) A_{0}^{\prime}=-k_{c} a j_{0}^{\prime}\left(k_{c} a\right), \\
& \Gamma_{c} h_{0}\left(k_{c} a\right) A_{0}+b_{2} B_{0}-\Gamma_{c}^{\prime} j_{0}\left(k_{c}^{\prime} a\right) A_{0}^{\prime}=-\Gamma_{c} j_{0}\left(k_{c} a\right), \\
& q_{c n} A_{0}+b_{3} B_{0}-q_{c n}^{\prime} A_{0}^{\prime}=-q_{0 n},
\end{aligned}
$$

where some terms have been grouped together to define new parameters

$$
\begin{aligned}
& b_{1}=k_{T} a \frac{h_{0}^{\prime}\left(k_{T} a\right)}{h_{0}\left(k_{T} a\right)}\left(1-\frac{\tau \Gamma_{T}}{\tau^{\prime} \Gamma_{T}^{\prime}}\right), \\
& b_{2}=\Gamma_{T}\left(1-\frac{\tau}{\tau^{\prime}} \cdot \frac{k_{T} a}{k_{T}^{\prime} a} \cdot \frac{h_{0}^{\prime}\left(k_{T} a\right)}{h_{0}\left(k_{T} a\right)} \cdot \frac{j_{0}\left(k_{T}^{\prime} a\right)}{j_{0}^{\prime}\left(k_{T}^{\prime} a\right)}\right), \\
& b_{3}=q_{T n}-\frac{\tau \Gamma_{T}}{\tau^{\prime} \Gamma_{T}^{\prime}} \cdot \frac{k_{T} a}{k_{T}^{\prime} a} \cdot \frac{h_{0}^{\prime}\left(k_{T} a\right)}{h_{0}\left(k_{T} a\right)} \cdot \frac{j_{0}\left(k_{T}^{\prime} a\right)}{j_{0}^{\prime}\left(k_{T}^{\prime} a\right)} \cdot q_{T n}^{\prime} .
\end{aligned}
$$

The solution of Eqs. (49)-(51) is the scattering coefficient $A_{0}$. Since it is now only a $3 \times 3$ set of equations, an analytical solution becomes more feasible.

\section{Separating thermal effects}

The zero-order coefficient includes effects due to differences in compressibility and attenuation between the two materials, and those due to thermal differences. Since under some conditions the thermal contribution may be negligible, it is helpful to separate the thermal effects from the rest. When the thermal modes become very small, the matrix solution would be ill-conditioned since the equations will be nearly identical and the matrix nearly singular. This has clearly been a problem where a solution for the matrix boundary equations is forced where the thermal contribution is negligible.

The coefficient is expressed as a sum of nonthermal and thermal terms,

$$
A_{0}=A_{0 N}+A_{0 T}, \quad A_{0}^{\prime}=A_{0 N}^{\prime}+A_{0 T}^{\prime} .
$$

The subscripts $n N$ and $n T$ denote the nonthermal and thermal part of the $n$th order coefficient. The nonthermal terms can be found by neglecting the thermal wave modes in the boundary equations and solving only the boundary conditions for $u_{r}$ and $P_{r r}$, Eqs. (49) and (51), leading to the results

$$
\begin{aligned}
& A_{0 N}=\frac{\left[k_{c} a q_{c n}^{\prime} j_{0}^{\prime}\left(k_{c} a\right)-k_{c}^{\prime} a q_{0 n} j_{0}^{\prime}\left(k_{c}^{\prime} a\right)\right]}{\left[k_{c}^{\prime} a q_{c n} j_{0}^{\prime}\left(k_{c}^{\prime} a\right)-k_{c} a q_{c n}^{\prime} h_{0}^{\prime}\left(k_{c} a\right)\right]}, \\
& A_{0 N}^{\prime}=\frac{\left[k_{c} a q_{c n} j_{0}^{\prime}\left(k_{c} a\right)-k_{c} a q_{0 n} h_{0}^{\prime}\left(k_{c} a\right)\right]}{\left[k_{c}^{\prime} a q_{c n} j_{0}^{\prime}\left(k_{c}^{\prime} a\right)-k_{c} a q_{c n}^{\prime} h_{0}^{\prime}\left(k_{c} a\right)\right]} .
\end{aligned}
$$

The radial stress parameters $q_{c n}$ and $q_{c n}^{\prime}$ are dominated by viscous terms at low frequency when $\left|k_{S} a\right|$ is small [see Eqs. (26) and (27)]. In that region the nonthermal part of the zero-order coefficient is imaginary and thus the effect is almost entirely on velocity, with little contribution to attenuation. Epstein and Carhart's ${ }^{3}$ limiting analytical result in the long wavelength region is also imaginary. At higher frequencies, where $\left|k_{S} a\right|$ is large, the viscous terms are less significant in the radial stress parameter $q_{c n}$, which is dominated by the inertial contribution [the first terms in Eq. (26)]. Here there is some contribution to attenuation from the nonthermal coefficient. Equation (56) is the standard result for the scattering coefficient when thermal terms are neglected. Thermal effects are an additional contribution to this, but in some cases can be much larger than this nonthermal component. In other cases, the thermal term is negligible and the nonthermal result is the dominant part of the zero-order coefficient.

The remaining terms in the equations are solved by the thermal parts of the coefficients,

$$
\begin{aligned}
& k_{c} a h_{0}^{\prime}\left(k_{c} a\right) A_{0 T}+b_{1} B_{0}-k_{c}^{\prime} a j_{0}^{\prime}\left(k_{c}^{\prime} a\right) A_{0 T}^{\prime}=0, \\
& \Gamma_{c} h_{0}\left(k_{c} a\right) A_{0 T}+b_{2} B_{0}-\Gamma_{c}^{\prime} j_{0}\left(k_{c}^{\prime} a\right) A_{0 T}^{\prime}=c_{1}, \\
& q_{c n} A_{0 T}+b_{3} B_{0}-q_{c n}^{\prime} A_{0 T}^{\prime}=0,
\end{aligned}
$$

where

$$
c_{1}=-\Gamma_{c} j_{0}\left(k_{c} a\right)-\Gamma_{c} h_{0}\left(k_{c} a\right) A_{0 N}+\Gamma_{c}^{\prime} j_{0}\left(k_{c}^{\prime} a\right) A_{0 N}^{\prime} .
$$

The solution to this set of equations is straightforward

$$
A_{0 T}=\frac{c_{1}\left[\frac{b_{1}}{b_{3}} q_{c n}^{\prime}-k_{c}^{\prime} a j_{0}^{\prime}\left(k_{c}^{\prime} a\right)\right]}{D},
$$




$$
\begin{aligned}
D= & {\left[\left(\frac{b_{1}}{b_{3}} q_{c n}-k_{c} a h_{0}^{\prime}\left(k_{c} a\right)\right)\left(\frac{b_{2}}{b_{3}} q_{c n}^{\prime}-\Gamma_{c}^{\prime} j_{0}\left(k_{c}^{\prime} a\right)\right)\right.} \\
& \left.-\left(\frac{b_{1}}{b_{3}} q_{c n}^{\prime}-k_{c}^{\prime} a j_{0}^{\prime}\left(k_{c}^{\prime} a\right)\right)\left(\frac{b_{2}}{b_{3}} q_{c n}-\Gamma_{c} h_{0}\left(k_{c} a\right)\right)\right] .
\end{aligned}
$$

In summary, Eqs. (56), (61), and (62) are the solution to the ECAH scattering problem for the zeroth partial wave order in the limit $k_{c}^{2} / k_{T}^{2} \ll 1$ for any value of $k_{c} a$. The solution demonstrates the contribution from nonthermal effects through $A_{0 N}$ and thermal effects through $A_{0 T}$ independently. The nonthermal term is dependent only on $k_{c} a$ and $k_{c}^{\prime} a$ and includes no thermal factors. The thermal term is a function of the propagational wave numbers and the parameters $k_{T} a$ and $k_{T}^{\prime} a$ which relate to the thermal wave modes.

The new method avoids the use of a matrix solution which is nearly singular when the thermal contribution is small. The matrix solution requires higher and higher accuracy at large $k_{T} a$ when the thermal modes contribute ever smaller effects. At large frequency, where $k_{T} a$ is large, the thermal term decreases as $1 /\left(k_{T} a\right)$. However, the limiting analytical solution in this case is not of great use, other than to elucidate the nature of the decrease in magnitude of the thermal contribution at large frequency. Having included the Bessel function scaling factor in the thermal wave modes, it is possible to evaluate the thermal contribution for any $k_{T} a$ however large, by using the asymptotic values of the ratios such as $h_{0}^{\prime}\left(k_{T} a\right) / h_{0}\left(k_{T} a\right)$ as given in Sec. IV C. The problem of evaluating Bessel functions at large arguments is avoided. The separation of the thermal and nonthermal parts of the zero-order coefficient allows an estimate to be made of the significance of the thermal term. Previously, it was only possible to invert the boundary equation matrix to obtain the full coefficient, and the magnitude of the thermal effect was not separated. Here, an initial estimate of thermal effects can be made in order to determine whether or not thermal effects should be included in calculations for a particular system.

\section{B. Solution for higher orders $A_{n}, n>0$}

\section{Thermal contribution}

For higher orders, $n>0$, all of the six boundary equations must be satisfied. However, when $k_{c}^{2} / k_{T}^{2} \ll 1$, the thermal terms can be neglected in the velocity and stress equations. Epstein and Carhart ${ }^{3}$ pointed this out in Appendix A, when developing an approximate analytical solution for the long wavelength region for the first-order coefficient $A_{1}$. However, the assumption is also valid for any $k_{c} a$ and any order. These wider applications were not explored by Epstein and Carhart, and the simplifications resulting from the assumption are developed here.

The case for neglecting the thermal wave modes for orders $n>0$ is based on a comparison of Eqs. (42) and (47), and Eqs. (46) and (44). Within the frequency limit given, the ratio of the thermal factors for propagational and thermal modes is again small, Sec. II C. The pairs of similar equations imply that the contribution of the thermal modes in the nonthermal boundary equations (in velocity and stress) is negligible. Hence, for $n>0$ there remain only four boundary equations, those in $u_{r}, P_{r r}, u_{\theta}, P_{r \theta}$, with the thermal modes neglected. These can be written in the following form:

$$
\begin{gathered}
k_{c} a h_{n}^{\prime}\left(k_{c} a\right) A_{n}-n(n+1) C_{n}-k_{c}^{\prime} a j_{n}^{\prime}\left(k_{c}^{\prime} a\right) A_{n}^{\prime}+n(n+1) C_{n}^{\prime} \\
=-k_{c} a j_{n}^{\prime}\left(k_{c} a\right)-k_{T} a \frac{h_{n}^{\prime}\left(k_{T} a\right)}{h_{n}\left(k_{T} a\right)} B_{n}+k_{T}^{\prime} a \frac{j_{n}^{\prime}\left(k_{T}^{\prime} a\right)}{j_{n}\left(k_{T}^{\prime} a\right)} B_{n}^{\prime}, \\
q_{c n} A_{n}+q_{S n} C_{n}-q_{c n}^{\prime} A_{n}^{\prime}-q_{S n}^{\prime} C_{n}^{\prime}=-q_{0 n}-q_{T n} B_{n}+q_{T n}^{\prime} B_{n}^{\prime}, \\
h_{n}\left(k_{c} a\right) A_{n}-\left[1+k_{S} a \frac{h_{n}^{\prime}\left(k_{S} a\right)}{h_{n}\left(k_{S} a\right)}\right] C_{n}-j_{n}\left(k_{c}^{\prime} a\right) A_{n}^{\prime} \\
+\left[1+k_{S}^{\prime} a \frac{j_{n}^{\prime}\left(k_{S}^{\prime} a\right)}{j_{n}\left(k_{S}^{\prime} a\right)}\right] C_{n}^{\prime}=-j_{n}\left(k_{c} a\right)-B_{n}+B_{n}^{\prime}, \\
d_{c n} A_{n}-d_{S n} C_{n}-d_{c n}^{\prime} A_{n}^{\prime}+d_{S n}^{\prime} C_{n}^{\prime}=-d_{0 n}-d_{T n} B_{n}+d_{T n}^{\prime} B_{n}^{\prime} .
\end{gathered}
$$

The full solution for the viscous and inertial coefficient is obtained by the matrix solution of these equations.

If the thermal terms are neglected entirely, $B_{n}=B_{n}^{\prime}=0$ the resulting matrix equation for the nonthermal coefficient is as follows:

$$
=\left(\begin{array}{c}
A_{n N} \\
C_{n N} \\
A_{n N}^{\prime} \\
C_{n N}^{\prime}
\end{array}\right)=\overline{\bar{R}}_{n N},
$$

where

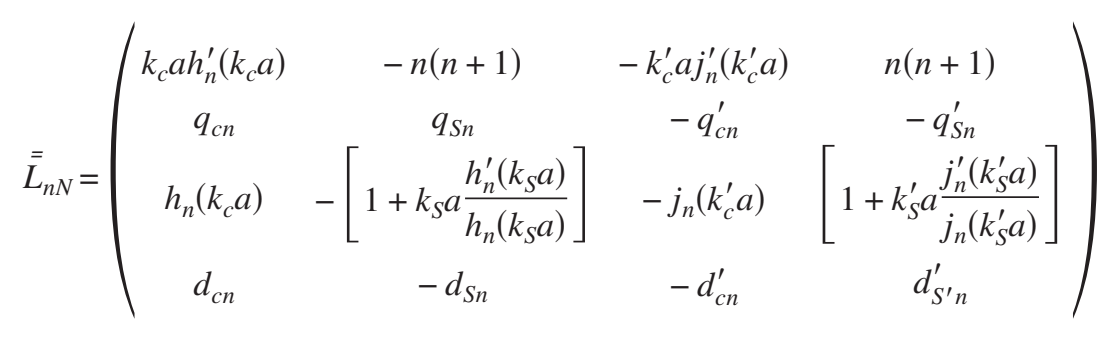

and 


$$
\overline{\bar{R}}_{n N}=\left(\begin{array}{c}
-k_{c} a j_{n}^{\prime}\left(k_{c} a\right) \\
-q_{0 n} \\
-j_{n}\left(k_{c} a\right) \\
-d_{0 n}
\end{array}\right)
$$

If thermal terms are neglected (setting $B_{n}$ and $B_{n}^{\prime}$ to zero), only four Eqs. (63)-(66) are solved, and the equations are much better conditioned numerically. Since the thermal terms were making a very small contribution, the full matrix equation was nearly singular. Removing the thermal terms has improved the conditioning on the matrix dramatically. In one example, the condition improved from $10^{10}$ to $10^{4}$, although the improvement depends on all parameters and in some cases, the matrix can still be badly conditioned after removing thermal terms. These conditions are discussed later.

In order to estimate the (initially neglected) thermal contribution, the calculated coefficients $A_{n N}$ and $A_{n N}^{\prime}$ (which have been obtained by neglecting thermal effects) can be substituted into the thermal boundary equations [Eqs. (46) and (47)], to estimate the thermal coefficients $B_{n}$ and $B_{n}^{\prime}$. The contribution of these thermal waves to the propagational mode coefficients can then be estimated by solving Eqs. (63)-(66) again, but with the estimated thermal contribution. Since the solution has already been obtained in the absence of thermal terms, the additional contribution to the coefficients, $\Delta A_{n T}$, etc., results simply from the extra terms on the right-hand side, so that

$$
=\left(\begin{array}{c}
\Delta A_{n T} \\
\Delta C_{n T} \\
\Delta A_{n T}^{\prime} \\
\Delta C_{n T}^{\prime}
\end{array}\right)=\Delta \overline{\bar{R}}_{n T}
$$

where

$$
\Delta \overline{\bar{R}}_{n T}=\left(\begin{array}{c}
-k_{T} a h_{n}^{\prime}\left(k_{T} a\right) / h_{n}\left(k_{T} a\right) B_{n}+k_{T}^{\prime} a j_{n}^{\prime}\left(k_{T}^{\prime} a\right) / j_{n}\left(k_{T}^{\prime} a\right) B_{n}^{\prime} \\
-q_{T n} B_{n}+q_{T n}^{\prime} B_{n}^{\prime} \\
-B_{n}+B_{n}^{\prime} \\
-d_{T n} B_{n}+d_{T n}^{\prime} B_{n}^{\prime}
\end{array}\right) .
$$

Thus the error in neglecting thermal effects can be easily estimated, to establish the validity of the approximation. The cycle could be repeated to obtain a more accurate result including thermal effects if necessary. First substitute the new estimate for the coefficients $A_{n}=A_{n N}+\Delta A_{n T}$ (and similarly for $A_{n}^{\prime}$ ) into the thermal equations (46) and (47), to obtain an improved estimate of the thermal coefficients, then solve Eq. (70) again to obtain a new estimate of the thermal contribution $\Delta A_{n T}$. The difference between consecutive estimates of the thermal contribution, $\Delta A_{n T}$, demonstrates whether the thermal effects are significant. When consecutive estimates are unchanged, the estimate is accurate. Note that the matrix inversion need only be carried out once, since it occurs in both equations with only the right-hand side being different.

The above-demonstrated method is of general validity where some terms in the equations make a small contribu- tion. The neglect of thermal terms in the boundary equations for orders $n>0$ was noted by Epstein and Carhart but applied only in a very limited set of conditions. Here, it is applied in the general case, and leads to a significant improvement in the condition of the matrix equation. In addition it is possible to estimate the error or thermal contribution, in order to establish confidence in the approximation, or to improve the calculation where necessary.

\section{Shear contribution}

At high frequencies, or at very low viscosity, when $\left|k_{S} a\right|^{2} \gg 1$, the shear mode terms make a negligible contribution and the viscous contribution to the stress components are negligible. These conditions are referred to as the inertial limit. When the shear modes contribute only a small amount in the boundary equations, the boundary matrix equation will again become nearly singular, and badly conditioned. It is therefore necessary under these conditions to separate the effect of the shear wave modes from the solution in a similar way to the separation of thermal terms in Sec. V B.1

The $n$th order coefficient can be expressed as

$$
A_{n N}=A_{n N I}+A_{n N V},
$$

where $A_{n N I}$ is the inertial limit and $A_{n N V}$ is the viscous contribution. The subscript $N$ denotes the fact that this is the nonthermal part of the solution, in which the thermal modes have been neglected. For the inertial limit, only the equations in radial velocity $u_{r}$ and stress $P_{r r}$ [Eqs. (42) and (43)] are relevant, with both the thermal and shear mode coefficients set to zero. The "density" or inertia terms dominate the stress components, and hence the viscous contributions $w_{0 n}, w_{c n}$. and $w_{c n}^{\prime}$ are neglected (Sec. IV B). Thus

$$
A_{n N I}=-\frac{\left[\hat{\rho} k_{c} a j_{n}^{\prime}\left(k_{c} a\right) j_{n}\left(k_{c}^{\prime} a\right)-k_{c}^{\prime} a j_{n}\left(k_{c} a\right) j_{n}^{\prime}\left(k_{c}^{\prime} a\right)\right]}{\left[\hat{\rho} k_{c} a h_{n}^{\prime}\left(k_{c} a\right) j_{n}\left(k_{c}^{\prime} a\right)-k_{c}^{\prime} a h_{n}\left(k_{c} a\right) j_{n}^{\prime}\left(k_{c}^{\prime} a\right)\right]}
$$

and inside the particle

$$
A_{n N I}^{\prime}=\frac{k_{c} a\left[j_{n}\left(k_{c} a\right) h_{n}^{\prime}\left(k_{c} a\right)-h_{n}\left(k_{c} a\right) j_{n}^{\prime}\left(k_{c} a\right)\right]}{\left[\hat{\rho} k_{c} a h_{n}^{\prime}\left(k_{c} a\right) j_{n}\left(k_{c}^{\prime} a\right)-k_{c}^{\prime} a h_{n}\left(k_{c} a\right) j_{n}^{\prime}\left(k_{c}^{\prime} a\right)\right]} .
$$

This is the true inertial limit. Although $A_{n N I}$ is a function of $k_{c} a$, the real part of $k_{c} a$ varies linearly with frequency, but the imaginary part varies with frequency in the same way as the attenuation, which could be second order or lower. Hence, when the attenuation is significant there may be some frequency dependence of $A_{n N I}$ which does not scale with $f a$.

Now it is necessary to determine the viscous or shear contribution to the coefficient $A_{n N}$ which is denoted $A_{n N V}$ [Eq. (72)]. In a similar way to the isolation of the remaining thermal terms in Sec. V B.1, the shear terms result from the matrix $L_{n N}^{=}$with the extra components on the right-hand side. Thus

$$
L_{n N}=\left(\begin{array}{c}
A_{n N V} \\
C_{n} \\
A_{n N V}^{\prime} \\
C_{n}^{\prime}
\end{array}\right)=R_{n N V}^{=}
$$




$$
R_{n N V}^{=}=\left(\begin{array}{c}
0 \\
-w_{0 n}-w_{c n} A_{n N I}+w_{c n}^{\prime} A_{n N I}^{\prime} \\
-(\hat{\rho}-1) j_{n}\left(k_{c}^{\prime} a\right) A_{n N I}^{\prime} \\
-d_{0 n}-d_{c n} A_{n N I}+d_{c n}^{\prime} A_{n N I}^{\prime}
\end{array}\right) .
$$

The resulting solution is exact (within the nonthermal approximation) using Eq. (72). However, the matrix equation which remains to be solved relies on inversion of the same matrix $L_{n N}^{=}$as did the full (nonthermal) solution. Hence it does not achieve any improvement in conditioning numerically, and the shear contribution $A_{n N V}$ becomes difficult to calculate at large values of $k_{S} a$. The only benefit in defining the problem in this way is to identify the contributions of inertial and viscous effects to the coefficient. The inertial part is the limiting solution at large frequencies.

In order to identify the behavior of the shear contribution as the inertial limit is approached, the solution for $A_{n N V}$ was obtained analytically and expanded as a series in powers of $1 /\left(k_{S} a\right)$. The appropriate limiting expressions for the Bessel functions at large arguments were substituted for $\left|k_{S} a\right|^{2} \gg 1$ and the parameter inside the particle defined as

$$
k_{S}^{\prime} a=\sqrt{\frac{\hat{\rho}}{\hat{\eta}}} k_{S} a .
$$

The algebraic solution was determined using MAPLE (version 10). The leading order term at large $\left|k_{S} a\right|$ was found to be

$$
\begin{aligned}
A_{n N V}= & -\frac{n(n+1)}{k_{c} a \cdot k_{S} a} \frac{\sqrt{\hat{\rho} \hat{\eta}}}{(1+\sqrt{\hat{\rho} \hat{\eta}})} \\
& \times\left[\frac{(\hat{\rho}-1) j_{n}\left(k_{c}^{\prime} a\right)}{\left[\hat{\rho} k_{c} a h_{n}^{\prime}\left(k_{c} a\right) j_{n}\left(k_{c}^{\prime} a\right)-k_{c}^{\prime} a h_{n}\left(k_{c} a\right) j_{n}^{\prime}\left(k_{c}^{\prime} a\right)\right]}\right]^{2} .
\end{aligned}
$$

However, the numerical results for all the terms in the series showed that the leading order term did not dominate under the conditions selected, and therefore should not be generally adopted as an approximate solution at large $\left|k_{S} a\right|$.

An alternative method is now proposed which is applicable when the shear wave modes cannot be neglected entirely, but their effect is so small that the matrix is becoming difficult to invert, such as happens at large $\left|k_{S} a\right|$ but before the inertial limit is reached. The method is similar to the approach taken to obtain an estimate of the thermal contribution, giving an approximate solution, with an estimate of the error.

In this case, a pseudoinertial limit is defined, by solving the $u_{r}$ and $P_{r r}$ boundary equations (42) and (43) with zero shear wave mode coefficients, but including the viscous parts of the stress components $w_{0 n}, w_{c n}, w_{c n}^{\prime}$. These are negligible in the true inertial limit. The coefficients are:

$$
A_{n N I S}=-\frac{\left[k_{c} a j_{n}^{\prime}\left(k_{c} a\right) q_{c n}^{\prime}-k_{c}^{\prime} a j_{n}^{\prime}\left(k_{c}^{\prime} a\right) q_{0 n}\right]}{\left[k_{c} a h_{n}^{\prime}\left(k_{c} a\right) q_{c n}^{\prime}-k_{c}^{\prime} a j_{n}^{\prime}\left(k_{c}^{\prime} a\right) q_{c n}\right]},
$$

$$
A_{n N I S}^{\prime}=-\frac{\left[k_{c} a j_{n}^{\prime}\left(k_{c} a\right) q_{c n}-k_{c} a h_{n}^{\prime}\left(k_{c} a\right) q_{0 n}\right]}{\left[k_{c} a h_{n}^{\prime}\left(k_{c} a\right) q_{c n}^{\prime}-k_{c}^{\prime} a j_{n}^{\prime}\left(k_{c}^{\prime} a\right) q_{c n}\right]} .
$$

In some cases, this value is a much better estimate of the full coefficient than is $A_{n N I}$ at frequencies below the true inertial limit. However, this does not appear to be generally true, but the pseudoinertial coefficient was used in this method as the initial estimate.

The contribution of the shear wave modes can be estimated by calculating $C_{n}, C_{n}^{\prime}$ by solving the boundary equations for tangential velocity $u_{\theta}$ and the $P_{r \theta}$ stress component [Eqs. (44) and (45)], given the values for $A_{n} \approx A_{n N I S}$ and $A_{n}^{\prime}$ $\approx A_{n N I S}^{\prime}$ presented earlier. Note that the thermal contribution is still neglected here. These values for $C_{n}, C_{n}^{\prime}$ can then be substituted back into the $u_{r}$ and $P_{r r}$ boundary equations (42) and (43) to determine the adjustment to $A_{n}$ caused by the shear wave modes. Using Eqs. (42) and (43) the estimated shear contribution to $A_{n}$ is as follows:

$$
\begin{aligned}
& \Delta R_{n N S 1}=n(n+1)\left(C_{n}-C_{n}^{\prime}\right), \\
& \Delta R_{n N S 2}=-q_{S n} C_{n}+q_{S n}^{\prime} C_{n}^{\prime}, \\
& \Delta A_{n N S}=\frac{\left[\Delta R_{n N S 1} q_{c n}^{\prime}-k_{c}^{\prime} a j_{n}^{\prime}\left(k_{c}^{\prime} a\right) \Delta R_{n N S 2}\right]}{\left[k_{c} a h_{n}^{\prime}\left(k_{c} a\right) q_{c n}^{\prime}-k_{c}^{\prime} a j_{n}^{\prime}\left(k_{c}^{\prime} a\right) q_{c n}\right]} \\
& \Delta A_{n N S}^{\prime}=\frac{\left[q_{c n} \Delta R_{n N S 1}-k_{c} a h_{n}^{\prime}\left(k_{c} a\right) \Delta R_{n N S 2}\right]}{\left[k_{c} a h_{n}^{\prime}\left(k_{c} a\right) q_{c n}^{\prime}-k_{c}^{\prime} a j_{n}^{\prime}\left(k_{c}^{\prime} a\right) q_{c n}\right]},
\end{aligned}
$$

and the estimated coefficient is given by

$$
A_{n N}=A_{n N I S}+\Delta A_{n N S} \text {. }
$$

The process can be reiterated to obtain an improved coefficient, by substituting the new coefficient back into Eqs. (44) and (45) to get a better estimate of the shear wave mode coefficients, followed by Eqs. (80)-(82) to find a more accurate value of $\Delta A_{n N S}$. When the matrix becomes difficult to invert, this method can be used to obtain an estimate for the shear terms, and iterated if necessary to obtain greater accuracy. Hence this is a valid and useful solution when the matrix becomes numerically badly conditioned or singular. The method removes the difficulty of ill-conditioned matrix equations, allowing solutions to be obtained under all conditions without resorting to extremely high precision programming. The use of the scaling with the Bessel function value at the particle surface has also removed the need to calculated Bessel functions at large values of $k_{S} a$ or $k_{S}^{\prime} a$ since only the ratios of derivatives are required. Limiting values for these have been presented in Sec. IV C.

\section{NUMERICAL RESULTS}

The solutions detailed in the previous sections have been programmed using MATLAB (version 7.1) under Windows XP and 64 bit precision, an accuracy of $2 \times 10^{-16}$. Calculations of the coefficients were carried out for a model emulsion of hexadecane in water (with $1 \%$ Tween20). The physical prop- 
TABLE I. Physical properties of $n$-hexadecane in water with $1 \%$ Tween 20 at $20{ }^{\circ} \mathrm{C}$ (Ref. 14).

\begin{tabular}{lcc}
\hline \hline & Water+1\% Tween20 & $n$-hexadecane \\
\hline Ultrasound velocity $\left(\mathrm{m} \mathrm{s}^{-1}\right)$ & 1485.5 & 1357.9 \\
Density $\left(\mathrm{kg} \mathrm{m}^{-3}\right)$ & 999.5 & 773 \\
Thermal expansivity $\left(\mathrm{K}^{-1}\right)$ & 0.00022 & 0.00091 \\
Viscosity (Pa s) & 0.00111 & 0.00334 \\
Specific heat capacity $\left(\mathrm{J} \mathrm{kg}^{-1} \mathrm{~K}^{-1}\right)$ & 4182 & 2093 \\
Thermal conductivity $\left(\mathrm{W} \mathrm{m}^{-1} \mathrm{~K}^{-1}\right)$ & 0.59 & 0.14 \\
Attenuation exponent $p$ & 2 & 2 \\
Attenuation factor $\left(\mathrm{Np} \mathrm{m} \mathrm{MHz}^{-1}\right)$ & 0.025 & 0.101 \\
\hline \hline
\end{tabular}

erties of the two components are given in Table I. ${ }^{14}$ Where relevant, the volume fraction was 0.1 .

\section{A. Zero-order coefficient $\boldsymbol{A}_{0}$}

The zero-order coefficient includes nonthermal and thermal contributions (see Sec. V A). The new solution has been compared with the ECAH solution for different particle sizes $0.1-10 \mu \mathrm{m}$ and frequency $0.01-1000 \mathrm{MHz}$. The match between the full ECAH matrix solution and the analytical results presented in Sec. V A was extremely accurate. The contribution of thermal effects is shown in Fig. 2 as a function of the thermal parameter $k_{T} a$. The plot covers a wide range of $k_{c} a$ values. For $a=0.1 \mu \mathrm{m}, k_{c} a=4 \times 10^{-6}-4$ and $\operatorname{Re}\left(k_{T} a\right)$ $=0.047-47$. For $a=10 \mu \mathrm{m}, k_{c} a=4 \times 10^{-4}-4, \operatorname{Re}\left(k_{T} a\right)=4.7$ -470. In this case, the coefficient is plotted in the form $A_{0 T} /\left(k_{c} a\right)^{3}$, which is the parameter appearing in velocity and attenuation. The plots for the two different particle sizes overlay for a large proportion of the range. However, they diverge at the higher frequency (large $\left|k_{T} a\right|$ ) end. In the long wavelength region, the parameter plotted $\left(A_{0 T} /\left(k_{c} a\right)^{3}\right)$ is purely a function of the thermal parameter $k_{T} a$, and therefore

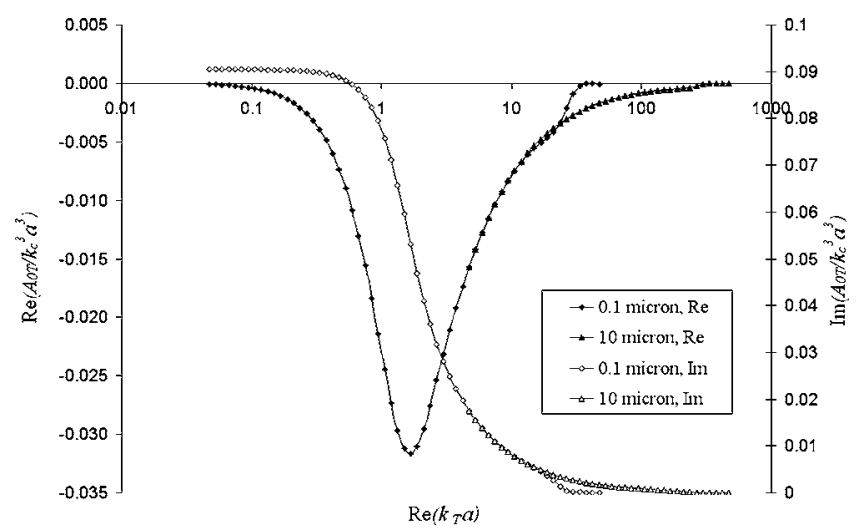

FIG. 2. Plot of the thermal part of the zero-order coefficient as a function of the thermal wave number radius product. This demonstrates the frequency dependence for two different particle sizes. The coefficient is scaled by $\left(k_{c} a\right)^{3}$ which is the term appearing in the wave number equation (20) for velocity and attenuation, and which removes the predominant frequency dependence in this range of $\left|k_{c} a\right|$ from $4 \times 10^{-6}-4$. The plots for different particle sizes overlay in the long wavelength region where $\left|k_{c} a\right| \ll 1$. The real part relates predominantly to the attenuation, and the thermal contribution decreases to zero at both low and high frequencies. The imaginary part relates to velocity and although the thermal contribution decreases to zero at high frequencies, it reaches a constant value at the low frequency limit, giving an offset in velocity.

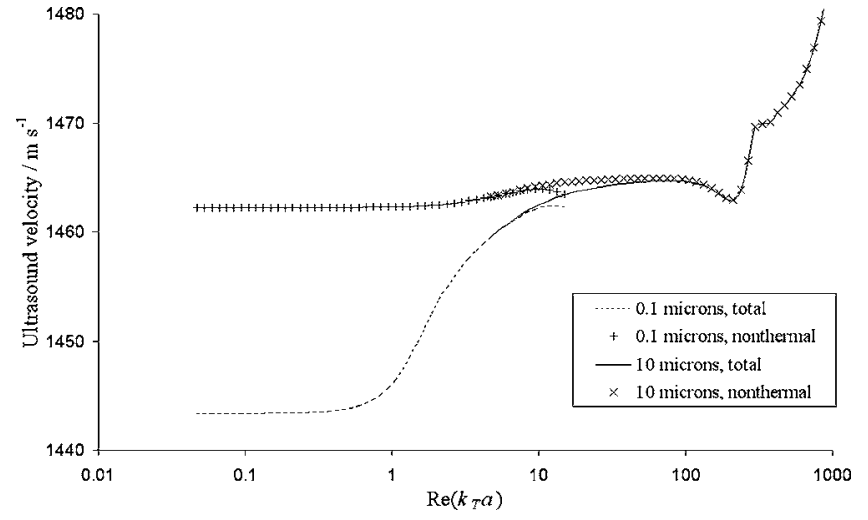

FIG. 3. Ultrasound velocity for a system of $10 \%(\mathrm{v} / \mathrm{v})$ hexadecane in water including or excluding the thermal contribution in the zero-order coefficient $A_{0}$. Higher order coefficients are included and have been calculated by the standard method, but the velocity is calculated using single scattering only (terms up to first order in volume fraction). The velocity is plotted as a function of the thermal parameter $\operatorname{Re}\left(k_{T} a\right)$ which demonstrates the frequency dependence for the different particle sizes. The plot covers the range $\left|k_{c} a\right|$ from $4 \times 10^{-6}-20$, and plots for the different particle sizes overlay where $\left|k_{c} a\right| \ll 1$. The thermal contribution to velocity becomes negligible at high frequencies and reaches a constant value at low frequency.

the results for different particle sizes (and therefore different $k_{c} a$ values) are the same. Outside that region, the value of $k_{c} a$ affects the result, and therefore the plots do not overlay. The point where the plots differ is the limit of the long wavelength region for the smallest particle size plotted.

Figure 2 shows that the real part of the thermal part of the coefficient decreases to zero at both ends of the frequency range, with a maximum around $\left|k_{T} a\right| \sim 1$. The real part of the scattering coefficient $A_{n}$ is the dominant contribution to attenuation, and the thermal effects are therefore negligible at both low and high frequency limits. However, the imaginary part of the thermal coefficient decreases to zero at the high frequency limit (large $\left|k_{T} a\right|$ ) but reaches a constant nonzero value as the frequency decreases (low $\left.\left|k_{T} a\right|\right)$. The imaginary part of the coefficient is the dominant contribution to the ultrasound velocity, resulting in an offset in velocity in the low frequency (or small particle size) limit. At high frequency, the thermal contribution to velocity is again negligible. It should be noted that the thermal coefficient is affected by the thermal wave number inside the particle as well as outside.

Solutions for the large values of $\left|k_{T} a\right|$ can easily be calculated in the new model. With the ECAH model, problems would be encountered for systems in which the particle size is large, and therefore $\left|k_{T} a\right|$ is large at experimental frequencies. Although the thermal contribution becomes negligible in the high frequency limit, the present method allows a means of estimating the thermal contribution without attempting to evaluate Bessel functions with large arguments, or solve an ill-conditioned matrix equation. As the thermal contribution becomes negligible the full matrix solution becomes nearly singular. It is now possible to estimate the thermal effect to demonstrate that it can be neglected.

The contribution of thermal effects to the ultrasound velocity and attenuation is shown in Figs. 3 and 4 for the radii of $0.1,1$, and $10 \mu \mathrm{m}$, and a frequency range of $0.01-1000$ $\mathrm{MHz}$, covering the range $\left|k_{c} a\right|$ from $4 \times 10^{-6}-20$. The result 


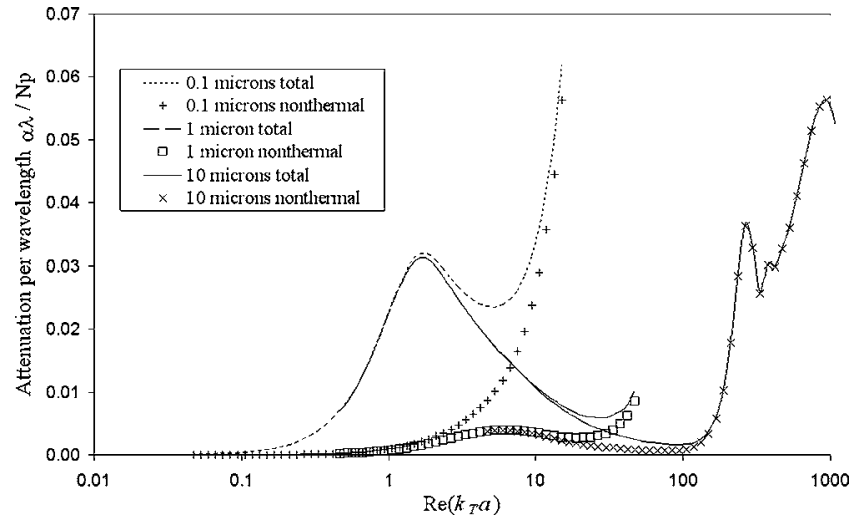

FIG. 4. Ultrasound attenuation per wavelength for a system of $10 \%$ hexadecane in water including or excluding the thermal contribution. Higher order coefficients are included and have been calculated by the standard method, but the attenuation is calculated using single scattering only (terms up to first order in volume fraction). The attenuation is plotted as a function of the thermal parameter $\operatorname{Re}\left(k_{T} a\right)$, which demonstrates the frequency dependence for the different particle sizes. The plot covers the range $\left|k_{c} a\right|$ from $4 \times 10^{-6}-20$. Plots for the different particle sizes overlay only where $\left|k_{c} a\right| \ll 1$ and the intrinsic attenuation is negligible. The thermal contribution to attenuation gives a peak in $\alpha \lambda$ and at large frequency [large $\operatorname{Re}\left(k_{T} a\right)$ ] the thermal contribution becomes negligible so that the nonthermal results approach the full result. The sharp increase in attenuation per wavelength at high frequencies is caused by the intrinsic attenuation (which varies as $f^{2}$ ).

for velocity and attenuation per wavelength $(\alpha \lambda)$ are again plotted as a function of $\operatorname{Re}\left(k_{T} a\right)$. Note that the attenuation per wavelength is the attenuation of the solution (using single scattering only) multiplied by the wavelength of sound in water. The plots include the results including or excluding the thermal part of the zero-order coefficient $A_{0}$. Higher order coefficients are included and have been calculated by the standard method, but the velocity is calculated using single scattering only (terms up to first order in volume fraction). The plots of velocity for the two particle sizes overlay in the long wavelength region as before. The features in the plot at high $\left|k_{T} a\right|$ for the large particle size occur when $k_{c} a \approx 1$ and the variation with frequency becomes complicated. Figure 3 shows that the thermal component causes a large shift in ultrasound velocity in the low frequency limit, but as the frequency (and $k_{T} a$ ) increases, the thermal contribution becomes negligible. The attenuation per wavelength plots overlay in the long wavelength region when the intrinsic attenuation contribution is negligible. An additional plot for $a$ $=1 \mu \mathrm{m}$ has been included to demonstrate the agreement of the lower part of the curves (small $\left|k_{T} a\right|$ ) for all particle sizes. However, as the frequency increases there are two reasons for the plots to differ for different particle sizes. First, the limit of the long wavelength region occurs at smaller values of $\left|k_{T} a\right|$ for smaller particle sizes, and outside this region there is dependence on $k_{c} a$ as well as the thermal parameter $\left|k_{T} a\right|$. Second, as the frequency increases, the intrinsic attenuation becomes significant causing the large increase in attenuation seen at around $\operatorname{Re}\left(k_{T} a\right) \approx 5$ for $a=0.1 \mu \mathrm{m}$, and $\operatorname{Re}\left(k_{T} a\right) \approx 200$ for $a=10 \mu \mathrm{m}$. Hence these attenuation plots do not overlay over the full range plotted here.

The thermal contribution to attenuation is negligible at both low and high frequencies (small or large $\left|k_{T} a\right|$ ), with a peak in the midrange. The peak occurs at around $\operatorname{Re}\left(k_{T} a\right)$ $\approx 1$. The strong increase in attenuation due to intrinsic attenuation truncates the thermal peak to a differing degree for each particle size. The nonthermal contribution to the attenuation per wavelength also has a peak, occurring at around $\operatorname{Re}\left(k_{T} a\right) \approx 6$, for the two larger particle sizes. This cannot be caused by thermal effects because it is in the nonthermal contribution to the scattering coefficient, but it varies with the thermal wave parameter $k_{T} a$. The peak is related to the maximum loss due to viscous and inertial effects, appearing through the stress parameter $q_{c n}$ and corresponding terms. These are determined by the shear wave parameter $k_{S} a$ which has the same frequency and particle size dependence as $k_{T} a$, and hence the two particle sizes show overlaying peaks.

\section{B. $A_{n} n>0$}

Calculations for orders $n>0$ were carried out for a system similar to hexadecane in water. It was required to determine the behavior of the shear contribution as a function of the parameter $k_{S} a$. This was carried out first for the same sets of parameters as used in the previous section, for hexadecane in water. However, a broader range of values of $k_{S} a$ and $k_{c} a$ was required in order to establish the general conditions under which the shear contribution can be neglected, or estimated. Calculations in which the frequency is varied for a given particle size result in large $k_{S} a$ when $k_{c} a$ is large, and vice versa, and the general case is not easily determined. Therefore a set of data was calculated for fixed values of $k_{c} a$, for each of which a range of values of $k_{S} a$ were used.

For each selected value of $k_{c} a$ a set of particle radius and frequency values were chosen to produce the relevant $\operatorname{Re}\left(k_{c} a\right)$ value, but for a range of $k_{S} a$ values. In order to achieve this, the viscosity in the two phases was adjusted, so that an appropriate range of $k_{S} a$ values could be obtained for very different $k_{c} a$ values. The parameters are limited by a number of conditions:

(1) The attenuation must be small compared with the real part of the wave number and

(2) The thermal limit is satisfied $\left|k_{c}^{2} / k_{T}^{2}\right| \ll 1$.

These are approximately satisfied for frequencies

$$
f \leqslant f_{\max } \approx 10 \mathrm{GHz}
$$

in water, and calculations were limited to this region. The smallest particle size corresponding to a given frequency with a set value of $k_{c} a$ is therefore

$$
a=\frac{\left(k_{c} a\right) v}{2 \pi f_{\max }} .
$$

Equation (86) defines a minimum particle size which can be used at the maximum frequency [Eq. (85)] to obtain a fixed value of $k_{c} a$. The shear wave parameter $k_{S} a$ is related by 
TABLE II. Viscosity values used for various values of $k_{c} a$.

\begin{tabular}{lll}
\hline \hline$k_{c} a$ & $\eta(\mathrm{Pa} \mathrm{s})$ & $\eta^{\prime}(\mathrm{Pa} \mathrm{s})$ \\
\hline 1 & 0.0111 & 0.0334 \\
5 & 1.11 & 3.34 \\
20 & 1.11 & 1.2 \\
50 & 30 & 35 \\
100 & 30 & 35 \\
\hline \hline
\end{tabular}

$$
\left|k_{S} a\right|=\sqrt{\frac{\omega \rho}{\eta}} a=k_{c} a v \sqrt{\frac{\rho}{2 \pi f \eta}}
$$

so at larger values of $k_{c} a$ the smallest value of $k_{S} a$ obtainable is limited by the maximum frequency. In order to explore lower $k_{S} a$ values, the viscosity has been increased appropriately. The values of viscosity used are given in Table II. All other properties for the system are those given in Table I.

\section{Thermal contribution}

In most cases the thermal contribution to the coefficients for orders $n>0$ can be neglected. The percentage error in the real and imaginary parts of the coefficients $A_{n}$ were calculated over a wide range of conditions. The percentage errors were found to be mostly less than $1 \%$, although in some cases a much larger error was found. These related to coefficients which were themselves small compared with the coefficients for other orders under the same conditions. The coefficients for different orders with the same parameters can be very different in magnitude, so that a large relative error in an order which has a very small coefficient can be tolerated. Conversely, a small relative error is required for the most significant coefficients. Hence the percentage error of individual coefficients was not a good measure of accuracy.

A measure of the significance of the coefficients to velocity and attenuation is obtained by comparison with the single scattering contribution

$$
\sum_{n}(2 n+1) A_{n} .
$$

The thermal contribution to this term is more relevant to velocity and attenuation calculations. The percentage error in the summation caused by neglecting thermal terms is mostly small $<1 \%$ in both real and imaginary parts, but there are instances where the error increases to a significant value, up to $5 \%$. The sum consists, in some cases, of a sum of large numbers of oscillating sign, which largely cancel out. This proves to be rather inaccurate. When there are a large number of orders to be included (when $k_{c} a$ is large) the factor $(2 n+1)$ amplifies any error in the largest orders so that the accuracy of these coefficients must be higher. Thus it is advisable to use the estimate of the thermal effect rather than neglecting the thermal effect altogether, to ensure the accuracy of the solution. Two iterations of the estimate are usually sufficient, but further iterations can be used until the required accuracy is achieved for the particular coefficient. Using the estimated thermal contribution, the solution was indistinguishable from the ECAH solution over the full range where calculation was possible.

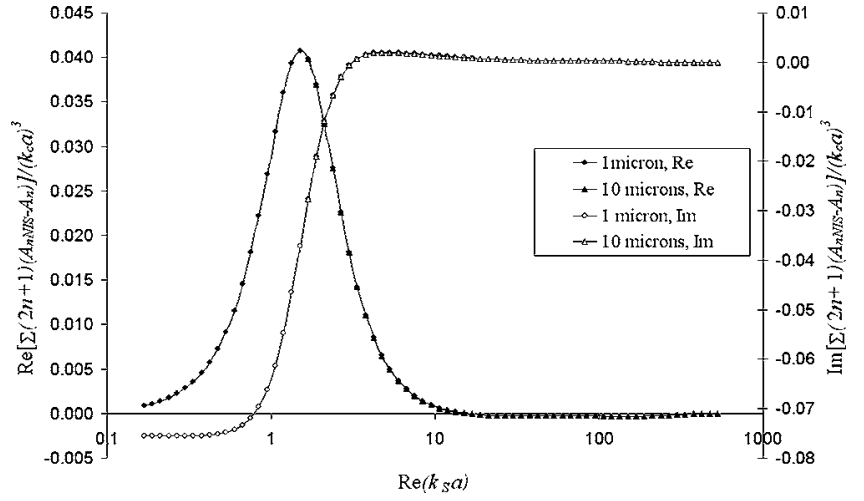

FIG. 5. The relative contribution of shear waves to the velocity and attenuation as a function of the shear parameter $\operatorname{Re}\left(k_{S} a\right)$, which shows frequency dependence for different particle sizes. The shear contribution is quantified by the difference in the sum $\Sigma_{n}(2 n+1) A_{n} /(k c)^{3}$ including or excluding shear waves. The imaginary part of this sum appears in the equation for the velocity of the dispersion and its real in the attenuation [Eqs. (21) and (22)]. The effect of shear waves on attenuation becomes negligible at both low and high frequency limits (the real part of the plotted difference becomes zero). At high frequency, the shear waves also make a negligible contribution to velocity (the imaginary part in the plot), but their effect reaches a constant value at the low frequency limit. The plot covers the range $\left|k_{c} a\right|$ from $4 \times 10^{-6}-40$ and plots for the different particle sizes overlay in the long wavelength region.

\section{Shear contribution}

The contribution made by the shear wave modes to the scattering coefficient was first investigated for the standard hexadecane in water system, whose properties are listed in Table I. Scattering coefficients were calculated for a number of particle sizes over a frequency range $0.01-100 \mathrm{MHz}$, either including or excluding shear wave modes from the calculation. The parameter used to demonstrate the significance of the shear wave contribution was again the sum $\Sigma_{n}(2 n+1) A_{n}$, taking the difference between the sum for the full coefficient $A_{n}$ and for the coefficient $A_{n N I S}$ which excludes shear wave modes. The real and imaginary parts of this shear wave contribution are plotted in Fig. 5 as a function of the shear wave parameter $\operatorname{Re}\left(k_{S} a\right)$. The real part of the shear wave contribution, which predominantly affects attenuation, falls to zero at both ends of the frequency range. The imaginary part, which predominantly affects ultrasound velocity, reaches a constant value as the frequency decreases, but at high frequencies its value falls to zero.

At large values of $\left|k_{S} a\right|$ the shear wave mode contribution, therefore, decreases to zero in both the real and imaginary parts of the coefficient (i.e., its effect on both velocity and attenuation vanishes at high frequency or for large particles). Hence the coefficient approaches the pseudo-inertial limit $A_{n N I S}$ at high frequencies. The true inertial coefficient $A_{n N I}$ is also approached when the viscous contribution to the stress terms [Eq. (26)] become negligible. As an example, Figs. 6(a) and 6(b) show the real and imaginary parts of a coefficient for $k_{c} a=20, n=5$, demonstrating the approach to the inertial limit. Also shown is the calculation estimating the shear wave contribution, using Eqs. (78) and (82), well before the inertial limit is reached, but when the shear effect is 


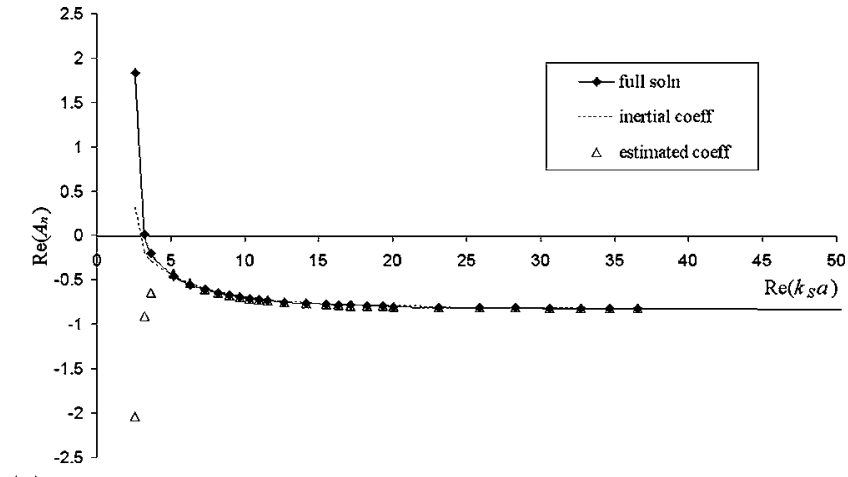

(a)

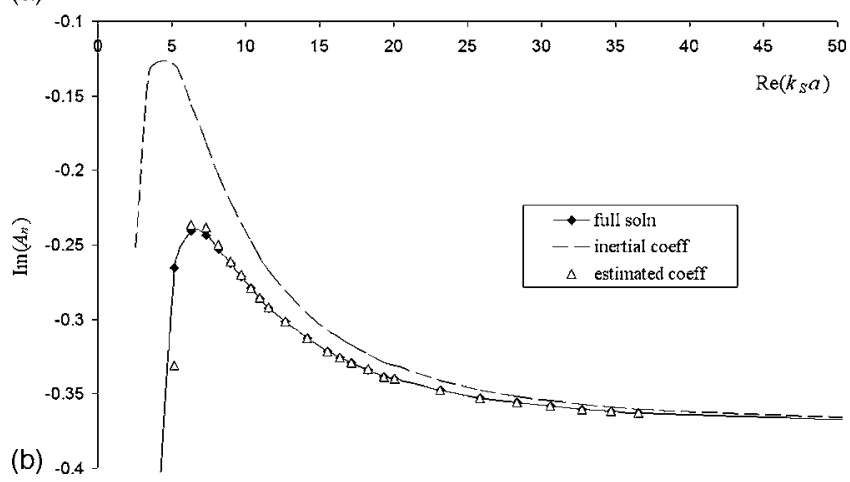

FIG. 6. The coefficient $A_{n}$ plotted as a function of $\operatorname{Re}\left(k_{S} a\right)$ for $k_{c} a=20$, $n=5$, showing the full calculations, the inertial limit and the coefficient calculated by estimating the small shear contribution when $\operatorname{Re}\left(k_{S} a\right)$ is large; (a) the real part (which predominantly affects attenuation) and (b) the imaginary part (which predominantly affects velocity). The plots demonstrate that at very large values of $\operatorname{Re}\left(k_{S} a\right)$ the coefficient approaches the inertial limit. However, the estimated shear contribution provides an accurate estimate of the coefficient at much lower values of $\operatorname{Re}\left(k_{S} a\right)$. The plot covers a range of particle size and frequency which combine to give a fixed value $k_{c} a=20$. Having fixed $k_{c} a$, an increasing value of $\operatorname{Re}\left(k_{S} a\right)$ corresponds to larger particle size, and lower frequency. The viscosity was set to be approximately $1000 \times$ its usual value in both phases (see the text for details) in order to cover a wide range of parameter space.

small. The estimate is poor at low values of $\left|k_{S} a\right|$ but shows very accurate agreement with the full coefficient at higher $\left|k_{S} a\right|$.

It is now necessary to determine the conditions under which the inertial limit is reached. For this purpose a set of calculations were carried out for a set of fixed values of $k_{c} a$, for each of which a range of values of $k_{S} a$ were calculated. The parameter $\Sigma_{n}(2 n+1) A_{n}$ was calculated, taking the difference between the full coefficient and the pseudoinertial limit $A_{n N I S}$, as for Fig. 5. This time, the difference is expressed as percentage error in the sum for the full coefficient. Figure 7 therefore shows the percentage error in the real and imaginary parts of the parameter $\Sigma_{n}(2 n+1) A_{n}$ caused by neglecting the shear wave modes. The percentage error approaches zero as $\left|k_{S} a\right|$ increases, but as $k_{c} a$ gets larger, the inertial limit is reached at a larger value of $\left|k_{S} a\right|$. It is also worth noting that the error is not monotonically decreasing, and a deceptively low value of the percentage error can be achieved at much smaller values of $\left|k_{S} a\right|$ than the true inertial limit, but the error subsequently rises again before falling to zero. This can be seen, for example in Fig. 7(a) for $k_{c} a=100$, which has a minimum when $\operatorname{Re}\left(k_{S} a\right) \approx 50$. However, the error does not finally fall below $1 \%$ until $\operatorname{Re}\left(k_{S} a\right) \geqslant 150$.
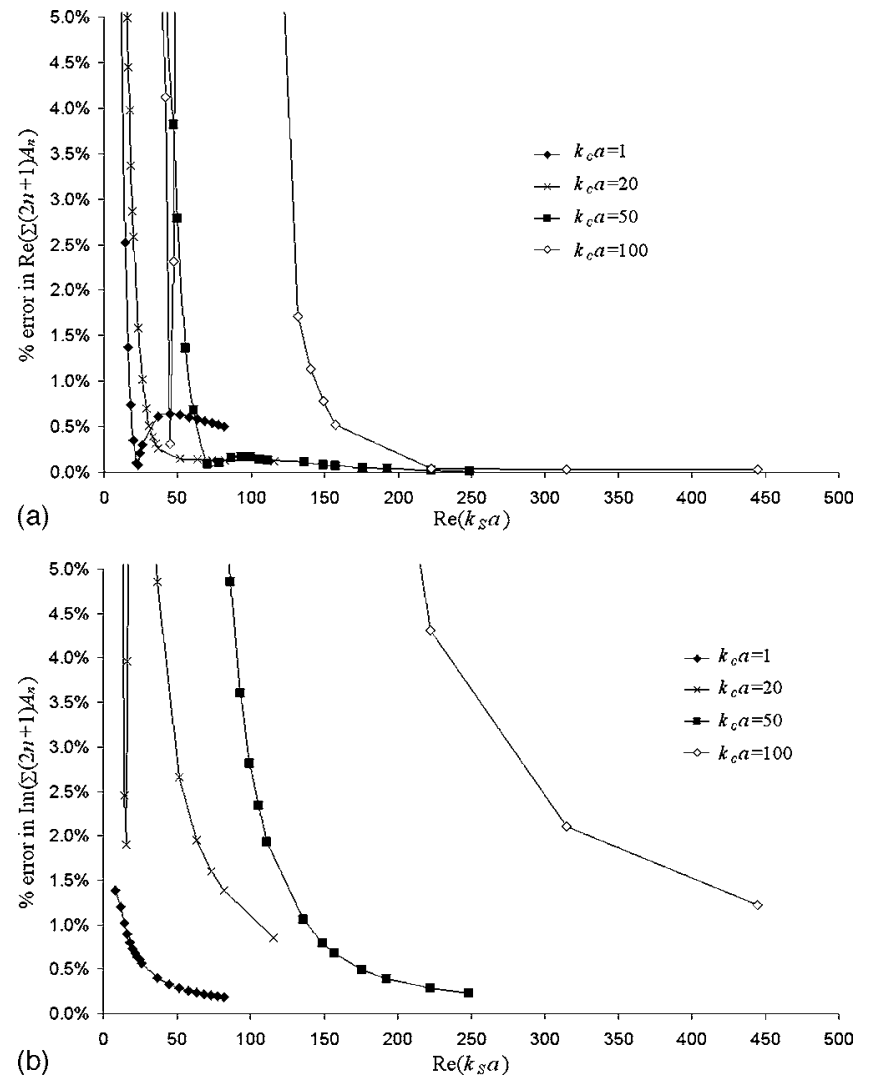

FIG. 7. The percentage error in the (a) real and (b) imaginary parts of the sum over coefficients $\Sigma_{n}(2 n+1) A_{n}$ calculated for the inertial limit and for the full coefficients. The real part of this sum relates to attenuation and its imaginary part to velocity. The purpose of the plot is to determine the limit at large $\operatorname{Re}\left(k_{S} a\right)$ at which the inertial coefficients become a satisfactory estimate for the full coefficients, i.e., when the plotted error becomes small. This limit is reached at larger values of $\operatorname{Re}\left(k_{S} a\right)$ when $k_{c} a$ is also large. The viscosity of the components was varied significantly to explore the parameter space.

An approximate relationship was obtained to define the inertial limit, where the error in both real and imaginary parts were found to be less than $1 \%$ for

$$
\left|k_{S} a\right| \geqslant 5 k_{c} a,
$$

when $5<k_{c} a<100$. The condition may be related to the relative contribution of inertial and viscous terms to the stress, Eqs. (26) and (27). This is a very approximate result, since only a few data points were obtained. Each data point requires the calculation of coefficients for a fixed value of $k_{c} a$ over a large range of $\left|k_{S} a\right|$ to identify the condition. When $k_{c} a$ is large, the shear effects continue to make a significant contribution for very large values of the shear parameter $\left|k_{S} a\right|$. Hence the methods presented here both for calculating the Bessel function ratios at large arguments and for estimating the shear contribution when it is very small are extremely valuable.

\section{CONCLUSIONS}

A number of methods have been presented which ease the numerical calculation of scattering coefficients in the ECAH model. First, a straightforward scaling of the thermal and shear wave modes by the respective Bessel or Hankel 
function (Heine function) evaluated at the boundary caused a significant improvement in the conditioning of the boundary equations. The scaling also removed the need to calculate directly the Heine functions at large arguments, since only ratios of the function derivatives with the function itself then appeared in the boundary conditions. Limiting values of these ratios at large arguments have been evaluated.

Further improvement of the solution was achieved by noting that under the condition $\left|k_{c}^{2} / k_{T}^{2}\right| \ll 1$ the thermal terms can be neglected in some of the boundary equations. The approximation is valid for frequencies up to around $100 \mathrm{GHz}$ in water. The fact that the thermal terms are negligible in some boundary equations implies their inclusion would cause near-singularity. For the zero-order coefficient, the use of this approximation, and the separation of the coefficient into thermal and nonthermal contributions produced an analytical result for the coefficient. Thus the thermal contribution can be readily estimated to determine whether it is significant for the system of interest. For the higher order coefficients the neglect of thermal terms improves matrix conditioning dramatically because the thermal contribution is small. A method is also presented for estimating the magnitude of the neglected thermal contribution either to improve accuracy or to confirm the validity of the approximation.

For the nonzero orders, even after thermal terms are neglected, the boundary equations can still become badly conditioned at large frequencies. In this region, the shear wave modes become insignificant, and this causes near-singularity of the matrix. The coefficient was separated into the inertial part (which is the limiting high-frequency value) and an additional shear contribution. This contribution can be estimated in a similar way to the thermal estimate. Thus the coefficient can be obtained in cases where previously the matrix solution was badly conditioned. It has been demonstrated that the inertial limit is reached at higher values of $\left|k_{S} a\right|$ (the shear wave parameter) when the value of $k_{c} a$ is large. Thus it may be necessary to evaluate the shear contribution for large values of $\left|k_{S} a\right|$ when $k_{c} a$ is also large since the shear contribution is still non-negligible. The method for calculating the Bessel/Hankel functions at large arguments are particularly relevant here.

In summary, the work presented here has achieved a number of improvements in the calculations for the ECAH scattering model. The conditioning of the matrix equations has been improved by introducing scaling factors for the thermal and shear wave potentials, and by neglecting thermal and shear mode contributions where appropriate. Further improvement has been obtained by developing a method for calculating the required Bessel functions. For the zero-order coefficient an analytical result has been obtained with separate nonthermal and thermal parts. These developments enable the model to be adopted on standard precision computers, rather than as a research tool on high precision machines, and potentially to be incorporated within instrumentation. Previously, such implementation has been limited to the long wavelength region where analytical results were available.

\section{ACKNOWLEDGMENT}

The author would like to thank Professor Malcolm Povey of the University of Leeds for his support of this work and for helpful comments on the manuscript.

\section{NOMENCLATURE}

$$
\begin{aligned}
& A, B, C=\text { scattering coefficients of compressional, } \\
& a=\text { particle radius } \\
& b_{1}, b_{2}, b_{3}, c_{1}=\text { parameters for the analytical solution of the } \\
& d=\text { scaled stress components } P_{r \theta} \\
& f=\text { frequency } \\
& f(\theta)=\text { far-field angular distribution of sound field } \\
& j_{n}, h_{n}=\text { spherical Bessel and Hankel functions, re- } \\
& \text { spectively, of the } n \text {th order } \\
& k_{c}, k_{T}, k_{S}=\text { wave numbers of compressional, thermal, } \\
& \text { and shear wave modes, respectively } \\
& K=\text { wave number of dispersion } \\
& \overline{\bar{L}}=\text { matrix to be inverted to obtain scattering } \\
& \text { coefficients } \\
& n \mathrm{Max}=\text { maximum number of partial wave orders re- } \\
& \text { quired for convergence } \\
& P_{n}, P_{n}^{1}=\text { Legendre polynomials, associated Legendre } \\
& \text { polynomials } \\
& P_{i j}=\text { components of stress tensor } \\
& q=\text { scaled stress components } P_{r r} \\
& \overline{\bar{R}}=\text { matrix for the right-hand side of the scatter- } \\
& \text { ing coefficient equation } \\
& T=\text { temperature } \\
& t=\text { time } \\
& \mathbf{u}=\text { (fluid) velocity } \\
& v=\text { sound speed (of compressional mode) } \\
& w=\text { scaled viscous contributions to stress com- } \\
& \text { ponent } P_{r r} \\
& \alpha=\text { attenuation (of compressional mode) } \\
& \beta=\text { thermal expansivity } \\
& \phi=\text { a scalar velocity potential; volume fraction } \\
& \varphi, \psi, \chi=\text { wave potential for compressional, thermal } \\
& \text { and shear modes respectively } \\
& \Gamma=\text { thermal factor } \\
& \gamma=C_{p} / C_{v}=\text { ratio of specific heat capacities } \\
& \eta=\text { shear viscosity } \\
& \hat{\eta}=\eta^{\prime} / \eta=\text { ratio of viscosities } \\
& \lambda=\text { wavelength } \\
& \rho=\text { density } \\
& \hat{\rho}=\rho^{\prime} / \rho=\text { ratio of densities } \\
& \sigma=\tau /\left(\rho C_{p}\right)=\text { thermal diffusivity } \\
& \tau=\text { thermal conductivity } \\
& \hat{\tau}=\tau^{\prime} / \tau=\text { ratio of conductivities } \\
& v=\eta / \rho=\text { kinematic viscosity } \\
& \omega=\text { angular frequency }
\end{aligned}
$$

\section{Subscripts and superscripts}

$0=$ (on wave potentials) incident wave 
$c, T, S=$ compressional, thermal, shear wave mode, respectively

,$=$ dispersed phase (particle)

$j_{n}^{\prime}, j_{n}^{\prime \prime}, h_{n}^{\prime}, h_{n}^{\prime \prime}=$ first and second-order derivatives of Bessel or Hankel function, respectively

$n=$ partial wave order

$\hat{x}=$ ratio of dispersed phase to continuous phase for property $x$, e.g., $\hat{\rho}=\rho^{\prime} / \rho$

$I=$ inertial part (neglecting shear waves and viscous contributions to stress)

$I S=$ pseudoinertial part (neglecting shear waves but including viscous stress)

$N, T=$ nonthermal and thermal parts

$S=$ viscous contribution (in addition to pseudoinertial limit)

$V=$ viscous contribution (in addition to inertial limit)

soln $=$ relating to the solution

${ }^{1}$ R. E. Challis, M. J. W. Povey, M. L. Mather, and A. K. Holmes, "Ultrasound techniques for characterizing colloidal dispersions," Rep. Prog. Phys. 68, 1541-1637 (2005).

${ }^{2}$ J. W. Strutt (Baron Rayleigh), The Theory of Sound, 2nd ed. (Macmillan, London, 1896).

${ }^{3}$ P. S. Epstein and R. R. Carhart, "The absorption of sound in suspensions and emulsions. I. Water fog in air," J. Acoust. Soc. Am. 25, 553-565
(1953).

${ }^{4}$ J. R. Allegra, and S. A. Hawley, "Attenuation of sound in suspensions and emulsions: Theory and experiments," J. Acoust. Soc. Am. 51, 1545-1564 (1972).

${ }^{5} \mathrm{C}$. F. Ying and R. Truell, "Scattering of a plane longitudinal wave by a spherical obstacle in an isotropically elastic solid," J. Appl. Phys. 27, 1086-1097 (1956).

${ }^{6}$ R. E. Challis, J. S. Tebbutt, and A. K. Holmes, "Equivalence between three scattering formulations for ultrasonic wave propagation in particulate mixtures," J. Phys. D 31, 3481-3497 (1998).

${ }^{7}$ O. G. Harlen, M. J. Holmes, M. J. W. Povey, Y. Qiu, and B. D. Sleeman, "A low frequency potential scattering description of acoustic propagation in dispersions," SIAM J. Appl. Math. 61, 1906-1931 (2001).

${ }^{8}$ T. J. O'Neill, J. S. Tebbutt, and R. E. Challis, "Convergence criteria for scattering models of ultrasonic wave propagation in suspensions of particles," IEEE Trans. Ultrason. Ferroelectr. Freq. Control 48, 419-424 (2001).

${ }^{9}$ A. S. Dukhin and P. J. Goetz, Ultrasound for Characterizing Colloids: Particle Sizing, Zeta Potential, Rheology (Elsevier, Amsterdam, 2002).

${ }^{10}$ M. J. W. Povey, Ultrasonic Techniques for Fluids Characterization (Academic, San Diego, 1997).

${ }^{11}$ P. Lloyd and M. V. Berry, "Wave propagation through an assembly of spheres. IV. Relations between different multiple scattering theories," Proc. Phys. Soc. London 91, 678-688 (1967).

${ }^{12}$ P. C. Waterman and R. Truell, "Multiple scattering of waves," J. Math. Phys. 2, 512-537 (1961).

${ }^{13}$ J. G. Fikioris and P. C. Waterman, "Multiple scattering of waves. II. 'Hole correction' in the scalar case," J. Math. Phys. 5, 1413-1420 (1964).

${ }^{14}$ D. J. McClements and J. N. Coupland, "Theory of droplet size distribution measurements in emulsions using ultrasonic spectroscopy," Colloids Surf., A 117, 161-170 (1996). 